\title{
Listeria monocytogenes personalized cancer vaccines drive therapeutic immune responses to cancer derived neoantigens
}

Brandon Coder ${ }^{1}$, Olga Pryshchep ${ }^{2}$, Dipti Kelkar ${ }^{1}$, Elena Filippova ${ }^{1}$, Xiaoming Ju ${ }^{1}$, David Balli ${ }^{1}$, Cristina Mottershead ${ }^{1}$, Kim Ramos ${ }^{1}$, Nithya Thambi ${ }^{1}$, Zhiyong Cheng ${ }^{2}$, Bryan Vander Lugt ${ }^{2}$, Justin Lesch ${ }^{2}$, Xian Liư $^{2}$, Jason DeVoss ${ }^{2}$, Keegan Cooke², Siyuan Liu², Jinghui Zhan², Petia Mitchell², Daniel O Villarreal ${ }^{1}$, Sandra M. Hayes ${ }^{1}$, James A Johnston ${ }^{2}$, Robert Petit ${ }^{1}$, Hyewon Phee ${ }^{2 *}$, Michael F. Princiotta ${ }^{{ }^{*}}$

*co-corresponding authors

Affiliation:

${ }^{1}$ Advaxis Inc, Princeton, NJ

${ }^{2}$ Inflammation and Oncology, Amgen Research, Amgen Inc, South San Francisco, CA

Corresponding Authors:

Michael F. Princiotta, PhD

E-mail: princiotta@advaxis.com

Advaxis Inc, Princeton, NJ

Hyewon Phee, PhD

E-mail: hyewonp@amgen.com

Inflammation and Oncology, Amgen Research, Amgen Inc, South San Francisco, CA

\section{Key words}

Listeria cancer vaccine, ADXS-NEO, Neoantigen, mouse pre-clinical tumor model, MC38 


\begin{abstract}
Background: Recent advances in the field of cancer immunotherapy have identified $\mathrm{CD} 8^{+} \mathrm{T}$ cell responses against tumor-specific mutations as a key driver of tumor regression and overall survival. ADXS-NEO is a personalized Listeria monocytogenes $(\mathrm{Lm})$-based immunotherapy designed to target a patient's mutation-derived tumor-specific neoantigens. The objective of this study is to demonstrate the feasibility of using the ADXS-NEO platform to target tumor-specific point mutations and control tumor growth by generating neoantigen-specific T cell responses using a pre-clinical mouse tumor model.
\end{abstract}

Methods: Whole-exome sequencing of the MC38 mouse tumor cell line identified 2870 unique nonsynonymous mutations. The netMHCcons algorithm was used to predict 137 potential neoantigens. We validated 20 immunogenic neoantigens either by peptide immunization followed by ELISPOT or by the presence of $\mathrm{CD}^{+} \mathrm{T}$ cells recognizing the neoantigen peptide following checkpoint inhibitor treatment. Two ADXS-NEO vectors were constructed; Lm20, targeting 20 validated immunogenic neoantigens, and Lm19, targeting most of the non-validated NSMs.

Results: Both Lm19 \& Lm20 significantly slowed tumor growth in C57BL/6 mice compared to control. An accumulation of ADXS-NEO-specific TILs was observed in tumor bearing mice treated with either Lm19 or Lm20. Examination of the tumor microenvironment in Lm19 or Lm20 treated mice revealed a decrease in the frequency and absolute number of Tregs, TAMs, MDSCs, and PD1 ${ }^{\text {high }}$ exhausted CD8 ${ }^{+} \mathrm{T}$ cells as well as an increase in the frequency and absolute number of effector $\mathrm{CD} 8^{+} \mathrm{T}$ cells, relative to control.

Conclusion: ADXS-NEO is a potent immunotherapy capable of driving immune responses against tumorspecific mutations and leading to tumor control in mice. 


\section{Background}

Genetic alterations found in cancer cells, but not in non-malignant cells, that result in nonsynonymous somatic mutations (NSM) can drive anti-tumor T cell responses ${ }^{1-5}$. Cancer-reactive $\mathrm{CD}^{+}$or $\mathrm{CD}^{+} \mathrm{T}$ cells can recognize mutated (MT) epitopes (neoepitopes) from neoantigens presented in the context of major histocompatibility complex (MHC) class I or class II molecules, respectively ${ }^{6}$. CD8 ${ }^{+} \mathrm{T}$ cells recognizing immunogenic neoepitopes are activated and proliferate, but ultimately become exhausted and express high levels of PD-1 as a result of prolonged exposure to antigen ${ }^{7,8}$. Anti-PD-1 or anti-PD-L1 checkpoint inhibitor cancer immunotherapy aims to reverse this exhaustion by blocking the activity of checkpoint molecules such as PD- $1^{9}$. Indeed, recent clinical studies using checkpoint inhibitor therapy demonstrated that neoantigen burden is a critical and predictive biomarker for responses to checkpoint therapy ${ }^{1,10-12}$, suggesting $T$ cell responses to neoantigens may control cancer growth if checkpoint inhibitors are provided. However, checkpoint inhibitor trials have demonstrated limited clinical efficacy as a monotherapy ${ }^{14}$ suggesting combination with other immunotherapies will be necessary for optimal benefit.

While checkpoint inhibition may help expand pre-existing populations of T cells, responses mediated by newly generated T cells require priming to the appropriate tumor-specific target antigens in the correct immunologic context ${ }^{6,14,15}$. One method of eliciting de novo T cell responses is through immunization using therapeutic cancer vaccines, which have been in development over the past two decades but have had limited success ${ }^{17}$. However, recent Phase 1 clinical studies using neoantigen-targeting cancer vaccines to treat advanced stage melanoma have re-established the potential of therapeutic vaccines for use in immunotherapy ${ }^{17-21}$. The recent success of adoptive T cell therapies targeting patient-specific neoantigens supports this approach ${ }^{23}$.

There are many outstanding questions and challenges to overcome before cancer vaccines become a viable therapy. First, the anti-tumor activity of neoantigen-specific $\mathrm{CD} 8^{+}$vs $\mathrm{CD} 4^{+} \mathrm{T}$ cells needs 
to be clarified. Interestingly, two previous cancer vaccine studies reported a strong $\mathrm{CD}^{+} \mathrm{T}$ cell bias when either peptide or mRNA was used for antigen delivery ${ }^{19,20,23}$. While the roles of $\mathrm{CD}^{+} \mathrm{T}$ cells as providing help to $C D 8^{+} T$ cells for differentiation into memory cells by providing IL-2, and, also as contributing directly as cytotoxic effector $\mathrm{CD} 4^{+} \mathrm{T}$ cells ${ }^{24}$, designing cancer vaccines to generate $\mathrm{CD} 4^{+} \mathrm{T}$ cells targeting MHC class II epitopes would not seem to be the most efficient approach because with the inherent cytotoxic ability of $\mathrm{CD} 8^{+} \mathrm{T}$ cells to kill tumor cells. In addition, there is currently no algorithm that can reliably predict the immunogenicity of a neoepitope ${ }^{25,26}$. As such, the capacity of a cancer vaccine to deliver multiple antigenic targets becomes far more critical, as the more neoantigens the vaccine targets, the greater the probability of eliciting a neoantigen-specific T cell response.

We initiated a study to test the feasibility of using a bacterial vector-based cancer vaccine as an immunotherapy targeting tumor derived neoantigens. We evaluated the potential of using Listeria monocytogenes $(\mathrm{Lm})$ as a personalized cancer vaccine (PCV), known as ADXS-NEO, utilizing the highly attenuated LmddA strain ${ }^{27}$. LmddA-based vectors are bioengineered to secrete one or more tumorassociated antigens fused to a tLLO peptide, including the tLLO secretion signal, transcribed from an extrachromosomal bacterial plasmid ${ }^{28}$. The LmddA strain is attenuated by the irreversible deletion of the actA virulence factor, which inhibits bacterial spreading ${ }^{29} . L m$-based vectors are rapidly phagocytosed by host antigen presenting cells $(\mathrm{APC})^{31}$, which results in the activation and maturation of not only the APC but also other innate immune cells, including neutrophils, macrophages, natural killer (NK) cells, and $\gamma \delta \mathrm{T}_{\text {cells }}{ }^{30}$. Activation of innate immune cells is facilitated by the recognition of $\mathrm{Lm}$ specific PAMPs (pathogen-associated molecular patterns) and DAMPs (damage-associated molecular patterns), including TLR2, TLR9, TLR5, and activation of the STING pathway ${ }^{30,31}$. Additionally, Lm infection drives a robust $\mathrm{CD} 8^{+} \mathrm{T}$ cell response and establishment of $\mathrm{CD} 4^{+} \mathrm{T}$ cell dependent $\mathrm{T}$ cell memory ${ }^{31}$. Once inside the APC, the $L m$-based vector escapes the phagosome and enters the host cell cytosol, where it secretes the tLLO fusion proteins containing the targeted TAAs ${ }^{30}$, which are then 
available to the MHC class I antigen processing and presentation pathway. Bacteria that do not escape the phagosome will ultimately be degraded in the phagolysosome, where antigenic proteins enter the MHC class II antigen presentation pathway ${ }^{32}$. Simply stated, the live attenuated bacterial vector acts as a unique self-enclosed antigen delivery system, expressing tumor antigens in the context of the initial stages of a bacterial infection with multiple strong adjuvant properties that allows for the simultaneous activation of innate and adaptive immunity.

Here, we report the use of L. monocytogenes to target tumor-derived neoantigens. Using a preclinical cancer model, we demonstrate a potential PCV pipeline starting with patient tumor biopsy, wholeexome sequencing, neoantigen prediction and engineering and production of a baceterial vector (ADXSNEO) with the capacity to target upwards of 40 putative neoantigens per individual construct. We found that ADXS-NEO promotes a pro-inflammatory tumor microenvironment (TME), attenuates intratumoral immune suppression, and drives a robust neoantigen-specific cytotoxic T lymphocytes (CTL) response. This targeted inflammation inhibits tumor progression, extends overall survival, and confers long-lasting protective immunity.

\section{Methods}

\section{Comparative Whole Exome Sequencing (WES), RNA-seq, and data analysis}

\section{Next-generation sequencing}

Whole-exome sequencing libraries were constructed using the SureSelect Mouse all Exon Kit (Agilent). RNA sequencing libraries were constructed using the TrueSeq Stranded mRNA Sample Prep kit (Illumina). All sequencing was done on the Illumina HiSeqX platform.

Whole-exome and RNA sequencing, analysis and neoantigen identification

Paired-end reads were aligned to the mouse reference genome ( $\mathrm{GRCm} 38)$ using BWA-MEM aligner (version 0.7.15-r1140). Duplicate reads were removed from sorted alignment maps using Sentieon 
Dedup followed by indel realignment around known indels using Sentieon Realigner and base recalibration using Sentieon QualCal. Somatic variant analysis was performed using Sentieon TNseq Haplotyper algorithm (version 201711.02). Identified variants were annotated using Snpeff (version 4.3p). Alignment to the mouse reference genome ( $\mathrm{GRCm} 38)$ was performed using STAR aligner (version 2.4.1). Expression was determined using HTSeq (version 0.6.1). Read count data were normalized using DESeq2 (version 1.22.1). Somatic missense mutations were filtered for RNA expression (TPM $\geq 0.5)$ and potential MHC class I peptides with predicted binding affinities for mouse $\mathrm{H}-2 \mathrm{~K}^{\mathrm{b}}$ and $\mathrm{H}-2 \mathrm{D}^{\mathrm{b}}$ alleles $\leq 500$ $\mathrm{nM}$ were considered potential neoantigens. All sequencing and analysis performed at MedGenome.

\section{Mice and animal care}

Animal studies were performed at Amgen and Advaxis.

Amgen: All experimental studies performed at Amgen were conducted under protocols approved by the Institutional Animal Care and Use Committee of Amgen. Animals were housed at Association for Assessment and Accreditation of Laboratory Animal Care International-accredited facilities (at Amgen) in ventilated micro-isolator housing on corncob bedding. Animals had access ad libitum to sterile pelleted food and reverse osmosis-purified water and were maintained on a 12:12 hour light: dark cycle with access to environmental enrichment opportunities. Female C57BL/6 mice (Charles River Laboratories), 6 to 8 weeks of age were cared for in accordance with the "Guide for the Care and Use of Laboratory Animals, 8th Edition".

Advaxis: Female 8-12-week-old C57BL/6 (Jackson Labs \& Charles River Labs) were used in compliance with protocols approved by the Institutional Animal Care and Use committee of Advaxis, Inc. and in accordance with guidelines from the National Institutes of Health.

\section{Cell line}


MC38 cells were maintained in accordance with the Kerafast MC38 cell maintenance protocol. Cells were tested for mycoplasma every 12 months.

\section{Reagents}

SIINFEKL peptide (VACSIN) and CpG(tIr-1826-1) were from InvivoGen (San Diego, CA). Neoantigen peptides were purchased from LifeTein (Somerset, NJ) and BioSynthesis (Lewisville, TX). Dextramers were purchased from Immudex (Copenhagen, Denmark).

\section{Peptide immunization in naive mice}

Mice were immunized twice at a 7 day interval with either 9-mer or synthetic long peptides mixed with CpG intravenously (100 $\mu$ g peptide with $50 \mu \mathrm{g}$ CpG in $200 \mu$ I PBS per mouse). Splenocytes were harvested 7 days following the second immunization for immunological analysis. Amino acid sequences of the peptides used for immunization are shown in online supplementary table 1 . The sequences of three NeoAg synthetic long peptides used for the efficacy study (Fig.1e) are; Adpgk (GIPVHLELASMTNMELMSSIVHQQVFPT), Dpagt1 (EAGQSLVISASIIVFNLLELEGDYR), and Reps1 (GRVLELFRAAQLANDVVLQIMELCGATR). The sequence of the 27-mer MT SLP of Adpgk is TGIPVHLELASMTNMELMSSIVHQQVF.

\section{Screening of $\mathrm{T}$ cell responses recognizing minimal MHC class I binding neoantigen peptides derived}

\section{from neoantigens in checkpoint inhibitor-treated mice.}

MC38 cells were injected subcutaneously on the right flank of mice $\left(3 \times 10^{5}\right.$ cells) on study day 0 . Tumor volume $\left(\mathrm{mm}^{3}\right)$ was measured twice per week using electronic calipers. Once the tumors reached an average of approximately $50 \mathrm{~mm}^{3}$ (study day 7), animals were randomized into treatment groups (25 mice per group). Animals were then administered three intraperitoneal injections of anti-CTLA-4 (30 $\mu \mathrm{g} /$ mouse) and anti-PD-L1 (200 $\mu \mathrm{g} /$ mouse) or vehicle every third day. On day 15 spleens were 
harvested and pan-T cells were isolated as described and stored in liquid nitrogen. Screening to detect T cells recognizing minimal peptides from MC38 neoantigens was performed using a peptide restimulation mouse IFN $\gamma$ ELISpot assay (Cellular Technology Limited). Briefly, $1 \times 10^{5}$ pan-T cells were mixed with $3 \times 10^{5}$ antigen presenting cells (CD3-depleted splenocytes from naïve C57BL/6 mice) along with the minimal neoantigen peptides at a final concentration of $1 \mu \mathrm{mol} / \mathrm{L}$. The assay was incubated at $37^{\circ} \mathrm{C}$ for 18 hours and IFN $\gamma+$ spots were enumerated using a Fluorospot analyzer (Cellular Technology Limited). Anti-CTLA-4 (clone 9D9, mlgG2a) and anti-PD-L1 (clone MIH5, mlgG1) were produced internally at Amgen, Inc.

\section{Peptide immunization of MC38-implanted mice}

Six to eight-week-old C57BL/6 mice were implanted subcutaneously with MC38 tumor cells $\left(3 \times 10^{5}\right.$ cells per mouse). When tumors were palpable $\left(50-150 \mathrm{~mm}^{3}\right)$, mice were randomized and peptides with CpG (or PBS control) were administered intravenously, followed by booster immunization seven days later and tumor size was measured and spleens and tumors were harvested seven days following final immunization.

\section{IFN $\gamma$ ELISpot assays of splenocytes from immunized mice}

IFN $Y$ ELISpot assays were performed by coating plates overnight with antibody, washing with cRPMI once and blocking with $200 \mu$ l of cRPMI for 2 hours at room temperature. Half a million to one million splenocytes, or $1 \times 10^{5}$ pan-T cells from tumors, were plated per well in $100 \mu$ l of cRPMI. For splenocyte stimulation, $100 \mu \mathrm{l}$ of peptide was added at a final concentration of $10 \mu \mathrm{g} / \mathrm{ml}$. For pan-T stimulation, $3 \mathrm{x}$ $10^{5}$ antigen presenting cells (CD3-depleted splenocytes from naïve C57BL/6 mice) were added per well. Cells were incubated at $37^{\circ} \mathrm{C}$, and IFNy production was determined after $18 \mathrm{hrs}$ following manufacturer's instructions (551083, BD Biosciences).

\section{Dextramer staining}


For dextramer staining, a total of $1 \times 10^{6}$ splenocytes or $2 \times 10^{6}$ tumor cells were washed twice with FACS buffer (PBS, 2\% FBS, 2mM EDTA), stained with dextramers (Immudex) for 10 minutes at room temperature. Without additional washing, an antibody cocktail containing Fc block (14-0161-86, eBioscience) BV buffer (566349, BD Bioscience) were added. Cells were incubated at $4^{\circ} \mathrm{C}$ for 20 minutes, washed in FACS buffer, stained with fixable viability dye (65-0866-14, eBioscience) for 10 minutes at room temperature, washed with FACS buffer and flow cytometry was performed within 2 hours of final wash.

\section{Tumor re-challenge experiment}

For tumor re-challenge experiment, mice that had cleared tumor following vaccination and remained tumor-free $\geq 100$ days after tumor implantation and naïve age-matched control mice were implanted subcutaneously with $3 \times 10^{5}$ MC38 tumor cells. Tumors grew to palpable size in all naïve mice after 10 days, while mice that had cleared tumor did not show any sign of tumor growth. Spleens were harvested from all mice and immunophenotype analysis and ELISpot assay were performed.

\section{Flow cytometry and antibodies}

Flow cytometric analyses were done at either the Amgen Flow Cytometry Core Facility (South San Francisco) using an LSRII (BD) flow cytometer, or at Advaxis Inc. using an Attune Nxt (Thermo Fischer) flow cytometer. For surface staining, cells were incubated with fluorochrome-conjugated antibodies for 30 minutes at $4{ }^{\circ} \mathrm{C}$ using $1: 200$ dilutions of each antibody (unless otherwise specified). Cells were washed twice in FACS buffer prior to analysis or intracellular staining using the Foxp3/Transcription Factor Staining Buffer Set (eBioscience). Dead cells were excluded either using DAPI (Life technologies) or LIVE/DEAD ${ }^{\circledast}$ Fixable Dead Cell Stain Kits (Life technologies) or fixable viability dye (65-0866-14, eBioscience). Forward and side scatter was used to identify live lymphocytes. Data analysis was performed using FlowJo (version 9.6.2) software (Tree Star). 


\section{ADXS-NEO vector design}

Target sequences utilized in vector design are listed in online supplementary table 1. Briefly, the point mutation amino acid was flanked by 7-13 wildtype amino acids, e.g. XXXXXXXXXXXMXXXXXXXXXX, where $\mathrm{X}$ is the wildtype sequence and $\mathrm{M}$ is the mutant amino acid. Total length of the target sequence per NSM was 21 amino acids, except that the NSM was localized towards to the C-terminal end of the protein.

\section{Plasmid construction, DNA synthesis, ligation, subcloning, transformation, and vaccine production}

Target amino acid sequences were reverse-translated, and codon optimized for Listeria monocytogenes using OPTMIZER (genomes.urv.es/OPTIMIZER/), and DNA was synthesized. Insert DNA was ligated into the pUC57 shuttle vector and subsequently digested and ligated into the pAdv134 plasmid. ADXS-NEO plasmids were then separately transformed into electrocompetent $L$. monocytogenes strain LmddA to generate strains $\operatorname{Lm} 3, \operatorname{Lm} 19, \operatorname{Lm} 20$, and $\operatorname{Lm} 19+20$. The presence of the correct plasmid within each strain was verified by colony PCR specific for each NEO plasmid (data not shown).

\section{Immunization of MC38-impInated mice with $\operatorname{Lm} 19$ and $\operatorname{Lm} 20$}

ADXS-NEO vaccine was thawed from $-80^{\circ} \mathrm{C}$ in a $37^{\circ} \mathrm{C}$ heat block, pelleted at $14,000 \mathrm{rpm}$ for 2 minutes and supernatant was discarded. The pellet was then washed 2X with PBS and re-suspended in PBS to a final concentration of $1.5 \times 10^{8} \mathrm{CFU} / \mathrm{mL}$. MC38 tumor bearing mice were dosed intravenously with $200 \mu \mathrm{L}$ per mouse using a 29G syringe (BD \#324704) for a total of $3 \times 10^{7}$ CFU per mouse, once per week starting at day 10 post tumor implantation for a total of 3 doses, unless otherwise specified. 


\section{Results}

\section{Neoantigen identification in the MC38 tumor model}

The aim of this study was to test the feasibility of a comprehensive personalized cancer vaccine targeting NSMs identified using next-generation sequencing. We performed comparative whole-exome sequencing of the murine MC38 tumor cell line and C57BL/6 normal tissue. Out of 2870 NSMs identified, NSMs were prioritized based on RNA expression by selecting mutations with transcripts per million (TPM) $\geq 0.5$ based on RNA sequencing, which yielded a total of 972 NSMs (Fig. 1a-b). The NSMs were then filtered by predicted MHC class I binding affinity using the NetMHCcons algorithm. A total of 137 NSMs were predicted to bind $\mathrm{H}-2 \mathrm{~K}^{\mathrm{b}}$ or $\mathrm{H}-2 \mathrm{D}^{\mathrm{b}}$ with an IC50 $\leq 500 \mathrm{nM}$ and were considered potential neoantigens. Next, we screened the potential neoantigens predicted to bind MHC class I for their immunogenicity using two methods (Fig. 1a-d, Fig. 2a). As a first strategy, C57BL/6 mice were immunized with minimal peptides (9mer MT peptide) containing the mutated NSM and CpG as adjuvant. Seven days later, spleens were harvested from immunized mice and the presence of neoantigen-specific $\mathrm{CD}^{+} \mathrm{T}$ cells were evaluated by IFNy ELISpot. We screened 37 NSMs and detected neoantigen specific $\mathrm{CD}^{+} \mathrm{T}$ cell responses against 13 of these NSMs (Fig. 1c, online supplementary figure 1a-b, and online supplementary table 1).

As a second strategy to identify immunogenic NSMs, we utilized the fact that checkpoint inhibitor treatment can lead to the activation and proliferation of neoantigen-specific T cells. Therefore, we treated MC38 tumor bearing mice with anti-CTLA4 and anti-PD-L1 antibodies (CPI) and screened minimal peptides derived from 105 predicted NSMs. We identified 8 NSMs capable of elevating $\mathrm{CD}^{+} \mathrm{T}$ cell responses following anti-CTLA4 and anti-PDL1 inhibitor treatment (Fig. 1d left, online supplementary figure 1c). 
Synthetic long peptides (SLPs) of 21 to 27 amino acids in length have been used successfully as neoantigen vaccine approaches ${ }^{20,33}$. We generated SLP vaccines against 3 neoantigens (Adpgk, Reps1, Dpagt1) shown to have therapeutic efficacy in the MC38 tumor model by Yadav et $\mathrm{al}^{5}$. In contrast to the reported therapeutic efficacy of the SLP vaccines, the neoantigen SLP vaccines did not control tumor growth nor extended survival in MC38 tumor bearing mice compared to vehicle or CpG plus anti-CD40 adjuvant treatment alone (Fig. 1e). We then evaluated the identical long neoantigen sequences using a Listeria monocytogenes vector, which has been previously shown to be efficacious for tumor control when targeting long peptide sequences ${ }^{29}$. Listeria monocytogenes has been used as a cancer vaccine vector to target minimal epitopes and full-length peptides and is currently being evaluated in multiple clinical trials ${ }^{34}$. We generated the $L m d d a$-based $\operatorname{Lm} 3$ construct expressing the identical neoantigens in the aforementioned neoantigen SLP vaccine. We observed a significant reduction in tumor growth in MC38 tumor bearing mice treated with $\mathrm{Lm} 3$ as well as a significant increase in overall survival compared to vehicle or the ADXS-tLLO empty vector controls (Fig. 1f and 1i). To better characterize the CD8 ${ }^{+} \mathrm{T}$ cell response, we vaccinated mice with Adpgk SLP 27mer or minimal epitope 9mer MT peptides. The SLP vaccine against Adpgk did not increase the neoantigen-specific T cell response in the tumor compared to vehicle alone (Fig. 1g; online supplementary figure 2a lower panel). Interestingly, Lm3 was able to generate a significant intratumoral $\mathrm{CD}^{+} \mathrm{T}$ cell response to Adpgk relative to controls, which was similar to the minimal 9mer vaccine, even though Lm3 encodes for the long peptide (Fig. 1h). Collectively, these data suggest that the vaccine platform used to deliver antigen plays a crucial role in generating intratumoral immune responses and, ultimately, inhibiting tumor growth.

\section{Therapeutic efficacy of Listeria vectors targeting multiple neoantigens}

To evaluate the potential for $L m$ immunotherapy to generate neoantigen specific immune responses against multiple neoantigens and whether these responses lead to therapeutic efficacy, we constructed the Lm20 vector targeting 20 validated neoantigens (online supplementary table 1 ) identified in the 
MC38 tumor model. The 20 neoantigens were validated using the minimal peptide screening and checkpoint inhibitor approach described in Fig. 1, with 11 neoantigens identified from 9mer peptide immunization and 9 from checkpoint inhibitor treatment (Fig. 1a-d; Fig. 2a). In addition, we generated Lm19, targeting 18 neoantigens that failed to generate a positive IFNy response by in vivo peptide immunization and 1 neoantigen that generated a minimal partial response following CPI treatment (Fig.1c, Fig. 1d right, and online supplementary table 1b, far right). The Lm constructs were designed as a beads-on-a-string fusion protein with adjacent neoantigen target sequences joined by linker amino acid sequences and fused to the non-hemolytic tLLO sequence required for secretion of the fusion protein into the host cell cytosol (Fig. 2b). The Lm constructs are designed to incorporate missense mutations as 21mers to allow for processing of MHC class I and class II neoantigens (Fig. 2b). We evaluated the immunogenicity and therapeutic efficacy of $\operatorname{Lm} 19$ and $\operatorname{Lm} 20$ in MC38 tumor bearing mice. Robust responses against Adpgk and Copg1, and measurable responses against $>70 \%$ of targeted neoantigens were detected in the tumors following two doses of Lm20 (Fig. 2c). To our surprise, we were able to detect significant neoantigen-specific immune responses against $>50 \%$ of targeted neoantigens from the tumors of mice treated with Lm19 compared to control, even though the same neoantigens failed to generate detectable T cell responses using peptide vaccination (Fig. 2d). Furthermore, both Lm19 and Lm20 treatments slowed tumor growth, and Lm20 significantly prolonged survival, with approximately $30 \%$ of Lm20 treated animals achieving a complete response (Fig. 2e). Additionally, we evaluated the capacity of ADXS-NEO to express multiple neoantigen sequences by generating a single construct targeting all 39 neoantigens included in Lm19 and Lm20. We vaccinated mice using the Lm19_20 single construct or a combination of $\operatorname{Lm} 19+\operatorname{Lm} 20$. Both the Lm19_20 single construct and Lm19+Lm20 mixed vaccine significantly slowed tumor growth (online supplementary figure 3).

\section{Lm drives antigen-specific responses towards secondary neoantigens}


To monitor the immunogenicity of individual constructs during development of the bacterial vectors for these studies, we included SIINFEKL, a validated peptide sequence known to elicit robust $\mathrm{CD}^{+} \mathrm{T}$ cell responses in $\mathrm{C} 57 \mathrm{BL} / 6$ mice $^{35}$. Inclusion of this sequence had the added benefit of addressing the question of whether the presence of a strong antigenic peptide sequence would result in the attenuation of $\mathrm{T}$ cell responses to other peptide antigens in the vaccine vector and/or a reduction in the ability to control tumors in the MC38 tumor model. To determine the effect of an immunodominant epitope, SIINFEKL, on vaccine efficacy, we immunized MC38 tumor bearing mice with $L m$ constructs expressing either tLLO alone with no target antigen (ADXS-tLLO), a immunogenic SIINFEKL model antigen not expressed in the MC38 tumor line (ADXS-tLLO-SIINFEKL), or the validated neoantigens in Lm20 without SIINFEKL (Lm20*(without SIINFEKL)) or with SIINFEKL (Lm20) included in the construct. The inclusion of the SIINFEKL sequence in Lm20 did not diminish T cell responses to other antigenic peptide sequences in the vector. Both Lm20 and Lm20* (without SIINFEKL) showed the strongest levels of tumor control (Fig. 3a, online supplementary figure 4a-b), suggesting the inclusion of SIINFEKL in the vector did not affect its capacity to control tumor growth. Strong $\mathrm{CD} 8^{+} \mathrm{T}$ cell responses towards multiple neoantigens were produced from the tumors and spleens of Lm20 and Lm20* (without SIINFEKL)vaccinated mice (Fig. 3b and online supplementary figure 4c-d). Moreover, we found that ADXS-tLLOSIINFEKL vaccinated mice generated $\mathrm{CD} 8^{+} \mathrm{T}$ cell responses against Adpgk, Pfkl, Chac1 and Med12 even though these antigens were not included in the vector (Fig. 3b). We also observed modest levels of tumor growth inhibition in ADXS-tLLO-SIINFEKL vaccinated mice (Fig. 3c).

Both the ADXS-tLLO-SIINFEKL and Lm20 constructs induced $C D 8^{+} \mathrm{T}$ cell responses against the Adpgk, Pfkl, Chac1 and Med12 neoantigens in the tumor (Fig. 3b). However, T cell responses against Pfkl, Chac1, and Med12 neoantigens were abrogated in vaccinated mice with Lm20* (without SIINFEKL) vaccine when SIINFEKL was removed from the Lm20. These data suggest that the response to Pfkl, Chac1, and Med12 neoantigens in ADXS-tLLO-SIINFEKL and Lm20 vaccinated mice is dependent on the 
generation of a SIINFEKL response. Furthermore, $\mathrm{CD} 8^{+} \mathrm{T}$ cell responses against Adpgk and Copg1 from the tumor and spleen (Fig.3b and online supplementary figure 4c), and Dab2ip and Cpne1 from the spleen were maintained in Lm20*(without SIINFEKL), indicating that these responses are independent of generating a SIINFEKL response and specific to neoantigen-delivery.

Vaccination with ADXS-tLLO did not generate a significant response to neoantigens included in Lm20, although tumor growth was modestly inhibited when ADXS-tLLO was given at a higher dose (1e8 CFU/mouse), consistent with previous findings ${ }^{36}$ (Fig. 3 b-c). However, that does not preclude the potential for antigen-specific responses against other non-targeted MC38 neoantigens. We found that mice vaccinated with ADXS-tLLO were able to generate $C D 8^{+} \mathrm{T}$ cell responses towards neoantigens included in Lm19 (Extended Fig. 5a). The response varies between individual mice, thus we stratified ADXS-tLLO treated animals by tumor growth inhibition compared to PBS (responders vs nonresponders). The ADXS-tLLO responders have a significantly higher proportion of neoantigen reactive TILs (Extended Fig. 5b). These data demonstrate the induction of resopnses against non-targeted neoantigens is an intrinsic property of Listeria vaccination and may be further enhanced with the inclusion of immunodominant epitopes.

\section{ADXS-NEO drives a pro-inflammatory tumor microenvironment}

We next assessed the generation of effector $\mathrm{CD} 8^{+} \mathrm{T}$ cells in the TME of MC38 tumors following vaccination with ADXS-NEO (Fig. 4a and Online supplementary figure 6a). By Day 22, Lm19 and Lm20 vaccinated mice displayed early signs of tumor regression (online supplementary figure 6a). $\mathrm{Lm}$ immunization significantly increased the proportion of total TILs, CD8 ${ }^{+}$TILs, cytotoxic Foxp $3^{-} \mathrm{CD} 4^{+}$TILs, $\curlyvee \delta \mathrm{T}$ cells and terminally differentiated $\mathrm{KLRG} 1^{\mathrm{lo}} \mathrm{CD} 8^{+} \mathrm{T}$ cells in the tumor (Fig 4a; online supplementary figure $6 b-d)$. The observed TME changes were not the result of antigen specific responses but, rather, were associated with immunization with the tLLO expressing Lm vector. Interestingly, $\sim 40 \%$ of effector 
TILs in untreated MC38 tumor bearing mice are of the PD- $1^{\text {int/hi }}$ LAG- $3^{+}$exhausted phenotype concomitant with a reduction in the expression of the cytotoxic granule associated serine protease granzyme A (GzmA) (Fig. 4b-d). Treatment with $L m$ resulted in the emergence of a CD8 ${ }^{+}$PD- $1^{10}$ effector $T$ cell population with cytotoxic potential characterized by increased GzmA expression (Fig.4e-f). To determine if PD-1 expression is reduced in neoantigen specific T cells, we evaluated PD-1 expression on Adpgk-specific T cells. Although Adpgk-specific T cells emerge in non-vaccinated and empty vector vaccinated tumor-bearing mice, their PD-1 expression is significantly higher than mice vaccinated with constructs targeting the Adpgk neoantigen (Fig. 4g-h).

Immune evasion in cancer is facilitated by the recruitment of regulatory T cells (Tregs), myeloid derived suppressor cells (MDSCs) and tissue associated macrophages (TAMs) into the tumor, which correlates with disease progression and decreased overall survival ${ }^{37,38}$. We evaluated the suppressive TME landscape in ADXS-tLLO, Lm19 and Lm20 vaccinated MC38 tumor bearing mice and found that the frequency and total number of $\mathrm{CD} 4^{+} \mathrm{CD} 25^{\text {hi }} \mathrm{Foxp}^{+}$intratumoral Tregs was significantly reduced (Fig. 5ac), resulting in elevated effector CD8:Treg and effector CD4:Treg ratios ( Ext. Fig. 6e-f). Likewise, the mean fluorescence intensity of Foxp3 was markedly decreased in the intratumoral Tregs compared to Lm19- or Lm20- treated mice (Fig. 5d). Foxp3 expression on a per cell basis correlates with the suppressive capacity of Tregs, suggesting that the suppressive function of Tregs in $L m$ vaccinated mice may be attenuated. The disparity of Foxp3 expression and effector:Treg ratio between ADXS-tLLO and Lm19 or Lm20 suggests an antigen-specific modulation of intratumoral Tregs. The attenuation of Tregs following $L m$ treatment is confined to the TME and was not observed in the spleen or draining lymph node (online supplementary figure 7c-d). Additionally, the TAM compartment was substantially diminished following $\operatorname{Lm} 20$ treatment. We detected a decrease in the quantity and proportion of TAMs and MDSCs (online supplementary figure 8a-e). Furthermore, conversion of macrophages from the protumoral M2 (Arginase-I) towards the anti-tumoral M1 (iNOS) subset, characterized by both elevated 
iNOS production and reduced Arginase-I producing TAMs, was detected in all $L m$-treated mice (Fig. 5ef).

\section{ADXS-NEO therapy confers long-lasting and neoantigen-specific protective immunity}

The stochastic nature of thymocyte development generates TCR repertoire diversity that is not fully heritable between individual mice and, as a result, not all mice respond equally to $\mathrm{T}$ cell targeted therapies $^{39}$. We stratified responders and non-responders based on their respective tumor volume and growth curves (Fig. 6a) and evaluated T cells from the tumor and spleen. All responder mice had a higher proportion of $\mathrm{CD}_{4} 5^{+}$cells within the tumor, and harbored Adpgk-specific $\mathrm{CD} 8^{+} \mathrm{T}$ cells in the blood, spleen, and tumor, whereas non-responder mice showed Adpgk-specific CD8 ${ }^{+} \mathrm{T}$ cells only in the tumor (Fig. 6a,C). Responder mice generated a robust IFN $\gamma$ responses towards the Adpgk and Copg1 neoantigens, as well as MC38 tumor itself, while non-responder mice did not (Fig. 6b). Additionally, responder mice had lower PD-1 expression on Adpgk-specific TILs compared to non-responders (Fig. 6de). Furthermore, we observed complete tumor clearance in $20-30 \%$ of vaccinated mice (Fig. 7 a). Splenic T cells from tumor cleared (CR) mice contained high percentages of Adpgk-specific T cells (Fig. 7b) and generated robust $\mathrm{CD}^{+}$IFNy ${ }^{+}$responses when stimulated with Adpgk or Copg1 mutated peptides or total dissociated MC38 tumor (Fig. 7c). Additionally, CR mice have a higher proportion of $\mathrm{CD} 88^{+} \mathrm{CD} 107 \mathrm{a}^{+} \mathrm{T}$ cells, characteristic of a CTL phenotype associated with degranulation and tumoricidal capacity (Fig. 7d).

To determine if $L m$-generated neoantigen-specific T cell responses persist, we analyzed circulating CD8 ${ }^{+} \mathrm{T}$ cells in the blood of CR mice 100 and 200 days following tumor clearance. We observed a significantly higher proportion of circulating effector $\mathrm{CD} 8^{+} \mathrm{T}$ cells (Teff) (CD44 ${ }^{+} \mathrm{KLRg} 1^{+}$) and effector memory $\mathrm{CD} 8^{+} \mathrm{T}$ cells (Tem) $\left(\mathrm{CD} 44^{+} \mathrm{KLRG} 1^{-} \mathrm{CD} 62 \mathrm{~L}^{\mathrm{lo}} \mathrm{CD} 27^{+}\right)$in the $\mathrm{CR}$ mice compared to age matched naïve mice (Fig. 7e, top). We were also able to detect circulating Adpgk and Copg1 neoantigenspecific T cells in Lm20 treated CR mice 100 days after tumor clearance (Fig. 8e-f). Interestingly, >90\% of 
circulating Adpgk-specific T cells from the CR mice express phenotypic markers associated with effector T cells (CD44 ${ }^{\text {hi }}$ LRG $^{\text {hi }}$ ) (Fig. 7e). We next evaluated whether long-lived memory responses were protective by re-challenging CR mice with MC38 tumor cells. One mouse completely rejected rechallenge, and the other two mice cleared the tumor by day 21. (Fig. 7g). Concurrent with protective memory, tumor re-challenge lead to the recall and expansion of circulating neoantigen-specific T cells (Fig. 7f). Although Adpgk and Copg1 -specific $\mathrm{CD}^{+} \mathrm{T}$ cells were maintained at a very low level prior to rechallenge, their responses expanded three-fold 7 days after tumor implantation (Fig. 7f). 


\section{Discussion}

Neoantigen vaccines have the potential to lead the next wave of cancer immunotherapy, as both a monotherapy and alongside checkpoint inhibitors. The success of any neoantigen cancer vaccine will be predicated on its potential to overcome two major challenges: first to generate robust neoantigen-specific effector T cell responses with tumoricidal potential and second to convert a "cold" tumor microenvironment (TME) to "hot" by engaging both the innate and adaptive arms of the immune system. Here we establish, using a pre-clinical murine tumor model, that ADXS-NEO, a genetically engineered Listeria monocytogenes cancer vaccine, successfully overcomes these challenges. With the capacity to target upwards of 40 potential neoantigens per construct, we demonstrate that ADXS-NEO elicits robust T cell responses towards neoantigens in the MC38 tumor model. Moreover, because ADXSNEO is a live attenuated bacterial vector, systemic administration of the $L m$ vaccine drives proinflammatory innate and adaptive immunity and converts the "cold" TME to "hot," by attenuating intratumoral Tregs, MDSCs, and M2 TAMs and generating effector $\alpha \beta / \gamma \delta$ tumor infiltrating T cells and M1 macrophages. Altogether, these data identify ADXS-NEO as a promising personalized immunotherapy platform with a high capacity to drive effector T cell responses against multiple neoantigens while simultaneously engaging innate immune cells, ultimately producing robust and longlived immunity towards tumor derived neoantigens.

Recent advances in whole genome sequencing have enabled the rapid identification of nonsynonymous mutations from a relatively small tumor biopsy, making a truly personalized cancer vaccine possible ${ }^{21}$. The quantity of non-synonymous mutations (NSMs) are highly varied between tumor types, from potentially thousands in melanoma, lung and bladder cancers to single digits in prostate, glioblastoma, and various leukemias ${ }^{21}$. While a greater number of NSMs correlates with more neoantigen targets and an increased potential for anti-tumor immunity ${ }^{40}$, large numbers of NSMs also pose technical obstacles for the design and manufacture of cancer vaccines. The choice of which 
antigenic targets to include in a cancer vaccine is critical due to inherent limitations in vector capacity.

This makes antigenic prediction both a necessary step, as well as a potential limitation in the production of personalized cancer vaccines. Various algorithms have emerged with the goal of predicting the binding affinity of a potential neoantigen for a given $\mathrm{MHC}$ molecule. Even with improvements in our ability to predict peptide-MHC binding affinity, accurate neoantigen selection is further limited by our ability to predict TCR binding to the $\mathrm{pMHC}$ complex, and ultimately the capacity to generate a tumoricidal effector T cell response. Therefore, it is critical that any system used to deliver tumorspecific neoantigens for the purpose of eliciting anti-tumor T cell immunity be as efficient as possible with respect to the number of neoantigens successfully targeted.

For example, as shown in Figure 1, we observed that only 9 of 37 predicted tested neoantigens generated significant immune responses in all mice immunized using a minimal peptide + adjuvant approach. Furthermore, we showed that validated neoantigens were unable to control tumor growth when administered as a synthetic long peptide vaccine. Yet when these same validated neoantigens were delivered using the ADXS-NEO vector they were able to generate T cell responses that slowed MC38 tumor growth. Moreover, the ADXS-NEO Lm19 construct, targeting non-validated neoantigens that failed to elicit a strong IFN $\gamma$ response via peptide vaccination, not only generated neoantigen specific responses, but controlled tumor growth and extended overall survival. These findings highlight the disparity between the immunogenic potential of different vaccine delivery systems ${ }^{41}$, a potential that is further complicated by the route of immunization ${ }^{42}$.

Considering the limited effectiveness of the currently available predictive neoantigen algorithms, strategies for maximizing the effectiveness of personalized vaccines are paramount. One such strategy is to empirically validate potential neoantigens by screening patient PBMCs for neoantigen reactivity. However, this approach will miss neoantigens that fail to prime responses in the patient, either due to sub-optimal conditions present in "cold" TME's or the lack of sufficient antigenic protein 
for cross-presentation and priming by dendritic cells. Furthermore, empirical validation of individual neoantigens adds time to an already time constrained manufacturing process, making it of limited utility in treating patients in more advanced stages of disease. In the studies presented here, we demonstrate that neoantigen validation by minimal peptide immunization or by checkpoint inhibitor blockade, was not indicative of neoantigen immunogenicity when delivered by an intracellular $\mathrm{Lm}$-based vaccine, as Lm19 treated mice were able to generate T cells expressing IFN $\gamma$ in response to non-validated neoantigens.

Another strategy for overcoming the limitations of predictive neoantigen selection is to simultaneously target all or the majority of NSMs, thereby de-emphasizing the importance of accurate neoantigen prediction by increasing the quantity of potential neoantigens delivered by the vaccine (increasing the "shots on goal"). While certain high mutational load tumor types have hundreds of potentially targetable NSMs, most tumor types have fewer than 10 somatic mutations per Mb, which corresponds to $\sim 150 \mathrm{NSMs}$, and many have fewer than 1 somatic mutation per $\mathrm{Mb}^{3}$. Building on the "shots on goal" strategy for neoantigen vaccine design, we demonstrate that ADXS-NEO has the capacity to deliver upwards of 40 neoantigens per individual construct, with a potential to simultaneously immunize with multiple constructs while maintaining tumor control efficacy in the MC38 model (online supplementary figure 3). As NGS technology improves and multi-biopsy approaches emerge that may increase the quantity of variant calls ${ }^{43}$, cancer vaccines, such as ADXS-NEO, with the capacity to deliver many neoantigens simultaneously provides a platform that can address the unpredictability of neoantigen identification.

A pre-clinical study using an mRNA-based neoantigen vaccine reported that $\mathrm{CD} 4^{+} \mathrm{T}$ cells are required for tumor control, while $\mathrm{CD}^{+} \mathrm{T}$ cells play a minimal role ${ }^{44}$. Furthermore, early clinical data from an mRNA-based personalized cancer vaccine trial in melanoma found that the majority of vaccine induced antigen-specific $\mathrm{T}$ cell responses were $\mathrm{CD} 4^{+} \mathrm{T}$ cell rather than $\mathrm{CD} 8^{+} \mathrm{T}$ cell mediated, despite the 
use of an algorithm designed to predict MHC class I binding peptides for the identification of neoepitope sequences to include in the vaccine constructs ${ }^{19}$. In contrast to these findings, we showed that ADXSNEO induced a robust neoantigen-specific $C D 8^{+} \mathrm{T}$ cell response, likely due to the inherent bias towards $\mathrm{MHC}$ class I antigen presentation and generation of $\mathrm{CD} 8^{+} \mathrm{T}$ cell responses associated with $\mathrm{Lm}$ infection 31. However, ADXS-NEO also generates effector $\mathrm{CD} 4^{+}$helper $\mathrm{T}$ cell responses, allowing for the establishment of long-lasting memory and protection against tumoral relapse. Interestingly, we found circulating neoantigen-specific effector and effector memory T cells in the blood of ADXS-NEO treated mice more than 100 days after tumor clearance. Importantly, ADXS-NEO treatment preferentially generates a less exhausted PD-1 low population of neoantigen-specific TILs, and these PD-1 low TILs were shown to correlate with tumor control. Although neoantigen-specific TILs were detected in nonvaccinated and empty-vector vaccinated tumor-bearing mice, these TILs were PD-1 high. It is possible that PD-1 high T cells may be pre-existing, a phenomenon that may be related to T cell activation resulting from cross-presentation in non-vaccinated mice compared to direct MHC class I presentation in Lm20 treated mice. The skewing towards PD-1 low TILs and their overall contribution to tumor control, ability to establish a long-lived memory response, and overall survival warrants further investigation.

Our findings demonstrate that an $L m$-based personalized cancer vaccine can generate a strong effector and effector memory $\mathrm{CD} 8^{+} \mathrm{T}$ cell response able to control tumor growth and provide longlasting protection in mice. With a high capacity to target many neoantigens and the ability to maintain a pro-inflammatory tumor microenvironment, ADXS-NEO has the potential to overcome many of the obstacles impeding the success of personalized cancer vaccines. However, the overall efficacy of PCVs has yet to be established in the clinic. ADXS-NEO, along with many other PCV platforms, are currently being evaluated in phase I clinical trials. 


\section{Declarations}

\section{Ethics approval and consent to participate}

All data are generated using mice and there is no participant in this study.

\section{Consent for publication}

All authors agree with publication. The material presented in this submission has not been previously reported and is not under consideration for publication elsewhere.

\section{Availability of data and material}

All data are included in the manuscript and supplementary information.

\section{Competing interests}

B.D., D.K., E.F., X.J., D.B., C.M., K.R., D.V., R.P., and M.P. are current or former employees of Advaxis. O.P., Z.C., B.L., J.L., D.V., K.C., C.L., J.Z., P.M., and H.P. are employees of Amgen.

X.L. is a current employee of Pfizer. J.J is a current employee of A2 Biotherapeutics.

\section{Funding}

There is no funding agency involved in this study.

\section{Authors' contributions}

$\mathrm{BC}$ conceived, designed and performed experiments, analyzed data, and wrote the manuscript ; OP performed experiments and analyzed data; DP, EF, XJ, CM, KR, NT, ZC, BVL, JL, XL, JD, KC, SL, JZ, PM and DOV performed experiments and analyzed data; $\mathrm{DB}$ analyzed data; $\mathrm{SMH}, \mathrm{JAJ}$, and RP contributed the design of the study and analyzed the data; and HP and MFP conceived and designed the study, supervised the research, analyzed data, and wrote the manuscript.

\section{Acknowledgements}

We thank Jing Qing and Wenjun Ouyang for reading the manuscript and providing intellectual input. 


\section{References}

1. Hodi, S. F. et al. Improved Survival with Ipilimumab in Patients with Metastatic Melanoma. New Engl J Medicine 363, 711-723 (2010).

2. Hinrichs, C. S. \& Rosenberg, S. A. Exploiting the curative potential of adoptive T-cell therapy for cancer. Immunol Rev 257, 56-71 (2014).

3. Schumacher, T. N. \& Schreiber, R. D. Neoantigens in cancer immunotherapy. Science 348, 69-74 (2015).

4. Delamarre, L., Mellman, I. \& Yadav, M. Neo approaches to cancer vaccines. Science $\mathbf{3 4 8 , 7 6 0 - 7 6 1 ~}$ (2015).

5. Yadav, M. et al. Predicting immunogenic tumour mutations by combining mass spectrometry and exome sequencing. Nature 515, 572-576 (2014).

6. Heemskerk, B., Kvistborg, P. \& humacher, T. N. The cancer antigenome. Embo J 32, 194-203 (2013).

7. Wherry, J. E. T cell exhaustion. Nat Immunol 12, 492 (2011).

8. Jin, H.-T., Ahmed, R. \& Okazaki, T. Negative Co-Receptors and Ligands. Curr Top Microbio/ 350, 17-37 (2010).

9. Pauken, K. E. \& Wherry, J. E. Overcoming T cell exhaustion in infection and cancer. Trends Immunol 36, 265-276 (2015).

10. McGranahan, N. et al. Clonal neoantigens elicit T cell immunoreactivity and sensitivity to immune checkpoint blockade. Science (New York, N.Y.) 351, 1463-9 (2016).

11. Anagnostou, V. et al. Evolution of Neoantigen Landscape during Immune Checkpoint Blockade in Non-Small Cell Lung Cancer. Cancer Discov 7, 264-276 (2017).

12. Łuksza, M. et al. A neoantigen fitness model predicts tumour response to checkpoint blockade immunotherapy. Nature 551, 517 (2017).

13. Seidel, J. A., Otsuka, A. \& Kabashima, K. Anti-PD-1 and Anti-CTLA-4 Therapies in Cancer: Mechanisms of Action, Efficacy, and Limitations. Frontiers Oncol 8, 86 (2018).

14. Sánchez-Paulete, A. et al. Antigen cross-presentation and T-cell cross-priming in cancer immunology and immunotherapy. Ann Oncol 28, xii44-xii55 (2017).

15. Kratky, W., e Sousa, C., Oxenius, A. \& Spörri, R. Direct activation of antigen-presenting cells is required for CD8+ T-cell priming and tumor vaccination. Proc National Acad Sci 108, 17414-17419 (2011).

16. Melief, C., van Hall, T., Arens, R., Ossendorp, F. \& van der Burg, S. H. Therapeutic cancer vaccines. J 
Clin Invest 125, 3401-3412 (2015).

17. Carreno, B. M. et al. A dendritic cell vaccine increases the breadth and diversity of melanoma neoantigen-specific T cells. Science 348, 803-808 (2015).

18. Linette, G. P. \& Carreno, B. M. Neoantigen Vaccines Pass the Immunogenicity Test. Trends Mol Med 23, 869-871 (2017).

19. Sahin, U. et al. Personalized RNA mutanome vaccines mobilize poly-specific therapeutic immunity against cancer. Nature (2017) doi:10.1038/nature23003 .

20. Ott, P. A. et al. An immunogenic personal neoantigen vaccine for patients with melanoma. Nature (2017) doi:10.1038/nature22991 .

21. Schumacher, T. \& Schreiber, R. Neoantigens in cancer immunotherapy. Science 348, 69-74 (2015).

22. Tran, E. et al. T-Cell Transfer Therapy Targeting Mutant KRAS in Cancer. New Engl J Medicine 375, 2255-2262 (2016).

23. Kranz, L. M. et al. Systemic RNA delivery to dendritic cells exploits antiviral defence for cancer immunotherapy. Nature 534, 396-401 (2016).

24. Ostroumov, D., Fekete-Drimusz, N., Saborowski, M., Kühnel, F. \& Woller, N. CD4 and CD8 T lymphocyte interplay in controlling tumor growth. Cell Mol Life Sci 1-25 (2017) doi:10.1007/s00018-0172686-7.

25. Karasaki, T. et al. Prediction and prioritization of neoantigens: integration of RNA sequencing data with whole-exome sequencing. Cancer Sci 108, 170-177 (2017).

26. Teku, G. N. \& Vihinen, M. Pan-cancer analysis of neoepitopes. Sci Reports 8, 12735 (2018).

27. Shahabi, V., Mavey, Maciag, P., Rivera, S. \& Wallecha, A. Development of a live and highly attenuated Listeria monocytogenes-based vaccine for the treatment of Her2/neu-overexpressing cancers in human. Cancer Gene Therapy 53-62 (2010) doi:10.1038/cgt.2010.48 .

28. Chen, Z. et al. Episomal Expression of Truncated Listeriolysin O in LmddA-LLO-E7 Vaccine Enhances Antitumor Efficacy by Preferentially Inducing Expansions of CD4+FoxP3- and CD8+ T Cells. Cancer Immunol Res 2, 911-922 (2014).

29. Wallecha, A., Maciag, P., Rivera, S., Paterson, Y. \& Shahabi, V. Construction and Characterization of an Attenuated Listeria monocytogenes Strain for Clinical Use in Cancer Immunotherapy. Clin Vaccine Immunol 16, 96-103 (2009).

30. Wood, L. M. \& Paterson, Y. Attenuated Listeria monocytogenes: a powerful and versatile vector for the future of tumor immunotherapy. Frontiers Cell Infect Microbiol 4, 51 (2014).

31. Khan, S. H. \& Badovinac, V. P. Listeria monocytogenes: a model pathogen to study antigen-specific memory CD8 T cell responses. Semin Immunopathol 37, 301-310 (2015). 
32. Geginat, G., Schenk, S., Skoberne, M., Goebel, W. \& Hof, H. A Novel Approach of Direct Ex Vivo Epitope Mapping Identifies Dominant and Subdominant CD4 and CD8 T Cell Epitopes from Listeria monocytogenes. J Immunol 166, 1877-1884 (2001).

33. Castle, J. C. et al. Exploiting the Mutanome for Tumor Vaccination. Cancer Res 72, 1081-1091 (2012).

34. Flickinger, J. C., Rodeck, U. \& Snook, A. E. Listeria monocytogenes as a Vector for Cancer Immunotherapy: Current Understanding and Progress. Nato Adv Sci Inst Se 6, 48 (2018).

35. Fremont, D., Stura, E., Matsumura, M., Peterson, P. \& Wilson, I. Crystal structure of an H-2Kbovalbumin peptide complex reveals the interplay of primary and secondary anchor positions in the major histocompatibility complex binding groove. Proc National Acad Sci 92, 2479-2483 (1995).

36. Wallecha, A. et al. Listeria monocytogenes-Derived Listeriolysin O Has Pathogen-Associated Molecular Pattern-Like Properties Independent of Its Hemolytic Ability. Clin Vaccine Immunol 20, 77-84 (2013).

37. Evach, E. Fatal attraction: tumors beckon regulatory T cells. Nat Med 10, 900-901 (2004).

38. Gajewski, T. F., Schreiber, H. \& Fu, Y.-X. Innate and adaptive immune cells in the tumor microenvironment. Nat Immunol 14, 1014 (2013).

39. Derbinski, J., Pinto, S., Rösch, S., Hexel, K. \& Kyewski, B. Promiscuous gene expression patterns in single medullary thymic epithelial cells argue for a stochastic mechanism. Proc National Acad Sci 105, 657-662 (2008).

40. Chan, T. A. et al. Development of Tumor Mutation Burden as an Immunotherapy Biomarker: Utility for the Oncology Clinic. Ann Oncol Official J European Soc Medical Oncol (2018) doi:10.1093/annonc/mdy495 .

41. Rodriguez, F., Harkins, S., Slifka, M. K. \& Whitton, L. J. Immunodominance in Virus-Induced CD8+ TCell Responses Is Dramatically Modified by DNA Immunization and Is Regulated by Gamma Interferon†. J Virol 76, 4251-4259 (2002).

42. Belyakov, I. M. \& Ahlers, J. D. What Role Does the Route of Immunization Play in the Generation of Protective Immunity against Mucosal Pathogens? J Immunol 183, 6883-6892 (2009).

43. McGranahan, N. \& Swanton, C. Clonal Heterogeneity and Tumor Evolution: Past, Present, and the Future. Cell 168, 613-628 (2017).

44. Kreiter, S. et al. Mutant MHC class II epitopes drive therapeutic immune responses to cancer. Nature 520, 692 (2015). 


\section{Figure legends}

Figure 1. Immunogenicity screening of neoantigens from MC38 cancer cell line.

a, Illustration demonstrating the process of the neoantigen calling and validation. $\mathbf{b}$, Flowcharts of neoantigens identified at each stage of analysis and the number of neoantigens tested in immunogenicity screening and validated neoantigens. c, IFN $\gamma$ Elispot analysis using cells from spleens of mice following immunization of minimal mutated (MT) peptide (9mer peptide harboring mutated amino acid) ( $n=3$ per peptide), re-stimulated in the presence or absence of the minimal MT peptide. $d$, IFN $\gamma$ Elispot analysis re-stimulated with minimal MT peptides using splenic cells from MC38-implanted mice treated with Control or anti-CTLA4 and anti-PD-L1 (CPI treated). Shown are IFN $\gamma$ Elispot counts from restimulated conditions with minimal MT peptides after substracting the counts from the no peptide control. Left; Neoantigens showing significant differences in IFN $\gamma$ Elispot count between control and CPI treated; Right: Neoantigens show in some mice but not statistically significant due to variability of the IFN $\gamma$ Elispot count. e, Tumor volume measurement and survival study of the MC38-transplanted mice treated with either PBS, adjuvant (CpG plus anti-CD40) alone, and adjuvant (CpG plus anti-CD40) plus three synthetic long peptides encoding Adpgk, Dpagt1 and Reps1. f,i, Tumor volume measurement (f) and survival study (i) of the MC38-transplanted mice treated with ADXS-tLLO (ADXS-NEO expressing tLLO of listeriolysin, empty vector control) or Lm3 (ADXS-NEO expressing SLP sequences of Adpgk, Dpagt1 and Reps1 as a fusion protein of tLLO). g, IFN $\gamma$ Elispot analysis of pan T cells from the MC38 tumor immunized with synthetic long MT peptide $(27 \mathrm{mer}$ ) or minimal peptides (9mer) encoding Adpgk MT sequences. Re-stimulation was performed using Adpgk 9mer MT peptide. $\mathbf{h}$, IFN $\gamma$ Elispot analysis of pan-T cells from the MC38 tumor vaccinated with ADXS-tLLO or Lm3. Statistics, c-d, Unpaired t-test, *, $0.01<\mathrm{P}<0.05 ; * *, 0.001<\mathrm{P}<0.01 ; * * *, 0.0001<\mathrm{P}<0.001 ; * * * *, \mathrm{P}<0.0001$., n.s., not significant, $\mathrm{f}$, Two way ANOVA and Tukey multiple comparison, $* * *, 0.0001<\mathrm{P}<0.001 ; * * * *, \mathrm{P}<0.0001$. , g, Two way ANOVA for multiple comparison, $*, 0.01<$ Adjusted $\mathrm{P}<0.05$; ${ }^{* *}, 0.001<$ Adjusted $\mathrm{P}<0.01$. $\mathrm{h}$, Unpaired ttest, $*, 0.01<\mathrm{P}<0.05$, $\mathrm{i}$, Survival analysis Log-rank test $*, 0.01<\mathrm{P}<0.05$.

\section{Figure 2. Immunogenicity and efficacy of $\operatorname{Lm} 19$ and $\operatorname{Lm} 20$ in MC38 tumor model.}

a, Numbers of neoantigens tested in immunogenicity screening in vivo and validated neoantigens. $\mathbf{b}$, Illustration demonstrating the construct design of $\operatorname{Lm} 19$ and Lm20. c, IFN $\gamma$ Elispot analysis from tumors of the mice immunized with $\operatorname{Lm} 20$. d, IFN $\gamma$ Elispot analysis from tumors of the mice immunized with Lm19. e, Efficacy of Lm19 and Lm20 vaccination in MC38 model. Statistics, c-d, Unpaired t-test, *, 0.01< $\mathrm{P}<0.05 ; * *, 0.001<\mathrm{P}<0.01 ; * * *, 0.0001<\mathrm{P}<0.001 ; * * * *, \mathrm{P}<0.0001$., * compares to PBS, + compares to ADXS-tLLO, Two way ANOVA for treatment comparison*, $0.01<\mathrm{P}<0.05 ; * *, 0.001<\mathrm{P}<0.01 ; * * *$, $0.0001<\mathrm{P}<0.001 ; * * * *, \mathrm{P}<0.0001$. e, Two way ANOVA and Tukey multiple comparison $*, 0.01<\mathrm{P}$ $<0.05 ; * *, 0.001<\mathrm{P}<0.01 ; * * *, 0.0001<\mathrm{P}<0.001 ; * * * *, \mathrm{P}<0.0001$; Survival analysis Log-rank test *, $0.01<\mathrm{P}<0.05 ; * *, 0.001<\mathrm{P}<0.01 ; * * *, 0.0001<\mathrm{P}<0.001 ; * * * *, \mathrm{P}<0.0001$.

\section{Figure 3. Antigen spreading by ADXS-NEO}


$\mathbf{a}$, Top, Schema of the study; bottom, total cell numbers isolated from the tumor. $\mathbf{b}$, Representative ELISpot assay depicting the total number of IFNy ${ }^{+}$spots using ex-vivo pan T cells isolated from day 24 MC38 tumors in mice treated with either PBS or ADXS-tLLO, ADXS-tLLO-SIINFEKL, Lm20 and Lm20* (Lm20 without SIINFEKL). c, MC38 tumor volume kinetics in mice treated with either PBS, ADXS-tLLO ( $10^{8} \mathrm{CFU} /$ mouse), or ADXS-SIINFEKL ( $10^{8} \mathrm{CFU} /$ mouse). Statistics, $\boldsymbol{b}$, Two way ANOVA multiple comparison, * $0.01<$ Adjusted $\mathrm{P}<0.05 ; * *, 0.001<$ Adjusted $\mathrm{P}<0.01 ; * * *, 0.0001<$ Adjusted $\mathrm{P}<0.001$; $* * * *$, Adjusted $\mathrm{P}<0.0001$. c, Two way ANOVA and Tukey multiple comparison*, $0.01<\mathrm{P}<0.05 ; * *$, $0.001<\mathrm{P}<0.01 ; * * *, 0.0001<\mathrm{P}<0.001$.

Figure 4. ADXS-NEO generates a robust neoantigen-specific CTL response.

a-h, Day 22 harvested MC38 tumors, ( $n=4-7$ ) in PBS, ADXS-tLLO, Lm19, or Lm20 treated mice. a, Left to right: Immunophenotyping of total TILs, depicting an increase in total TILs, CD8 ${ }^{+}$TILs, memory CD8+ TILs, b. Representative flow cytometric immunophenotyping CD8 ${ }^{+}$effectors depicting a decrease in LAG-3 and PD-1 surface expression. c, Frequency of exhausted (PD-1 $1^{\text {int/hi } L A G-3^{+}}$) CD8 TILs, d, Loss of Gzma expression represented by the ratio of granzyme $A$ mean fluorescence intensity in exhausted (PD-

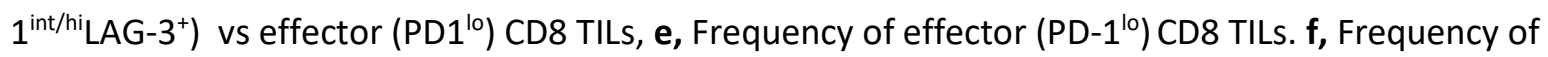
cytotoxic gzma+ effector CD8 TILs. g, Mean fluorescence intensity of PD-1 on Adpgk dextramer positive cells from CD8 TILs. $h$, Percentages of PD1 positive cells within Adpgk dextramer positive CD8 TILs. Statistics; a-f, Unpaired t-test, $*, 0.01<\mathrm{P}<0.05 ;{ }^{* *}, 0.001<\mathrm{P}<0.01 ; * * *, 0.0001<\mathrm{P}<0.001 ; * * * *, \mathrm{P}<$ 0.0001. g-h, One-way ANOVA for multiple comparisons. ${ }^{*}, 0.01<$ Adjusted $\mathrm{P}<0.05$; n.s., not significant.

\section{Figure 5. ADXS-NEO attenuates the suppressive tumor microenvironment.}

a-d, Flow cytometric phenotyping of the intratumor regulatory T cell compartment in day 22 harvested MC38 tumors in mice treated with ADXS-NEO, ( $n=4-7)$. e-f, Representative immunocytochemistry of day 22 harvested MC38 tumors. a, Gain of M1 phenotype (iNOS ${ }^{+}$, red) in TAMs (F4/80, green). b, Loss of M2 macrophages (Arg- $1^{+}$, red; F4/80 green) following treatment with $\mathrm{Lm}$. Statistics; Unpaired t-test, * $0.01<$ $\mathrm{P}<0.05 ; * *, 0.001<\mathrm{P}<0.01 ; * * *, 0.0001<\mathrm{P}<0.001 ; * * *, \mathrm{P}<0.0001$.

\section{Figure 6. Heterogeneous immune responses observed in ADXS-NEO treated mice.}

a, Tumor growth of responder and non-responder mice immunized with $\operatorname{Lm} 20 *(T o p)$. On day 36, mice with tumor size greater than $1200 \mathrm{~mm}^{3}$ were selected as "Non-responders" and mice with tumor size smaller than $500 \mathrm{~mm}^{3}$ were selected as "Responders." Bottom left, Total cell numbers from tumor, spleen and lymph nodes from Responder and Non-responder. Bottom right, Percentages of CD45+ cells from tumor of responder or non-responder mice. $\mathbf{b}$, Generation of tumor-specific IFN $\gamma$ from splenic CD8 T cells from responder or non-responder mice. c, Neoantigen-specific CD8 T cells in the blood and spleen of responder and non-responder mice. $\mathbf{d}$, Mean fluorescence intensity of PD1 on Adpgk dextramer positive cells from CD8 TILs of responders and non-responders. e, Percentages of PD1 positive cells 
within Adpgk dextramer positive CD8 TILs of responders and non-responders. Statistics, a, top and bottom left, Two-way ANOVA for multiple comparisons. *, $0.01<\mathrm{P}<0.05$; ****, $\mathrm{P}<0.0001$; bottom right, Unpaired t-test, ${ }^{*}, \mathrm{P}<0.05$. d-e, Unpaired t-test. ${ }^{*} 0.01<\mathrm{P}<0.05$.

\section{Figure 7. ADXS-NEO therapy confers long-lasting memory and neoantigen-specific protective immunity.}

a, Schema of the study (top). Bottom: Tumor growth(left) and survival (right) of the mice vaccinated with PBS $(n=10)$ or Lm20*(Lm20 without SIINFEKL, $n=10)$. b, Percentages of CD8 T cells recognizing mutated Adpgk assessed by Adpgk dextramer in the spleen of tumor-cleared mice 56 days after tumor implantation. CR, complete responder. c, Generation of IFN $\gamma$ from CD8 T cells from tumor-cleared mice re-challenged with irradiated MC38 or MT peptides of Adpgk or Copg1. Spleens from tumor-cleared mice were re-challenged with minimal peptides from MT Adpgk or Copg1 or irradiated MC38. d, CD107a upregulation following MC38 re-challenge. CD8 T cells from tumor-cleared mice induced CD107a when re-challenged with irradiated $\mathrm{MC} 38$, while CD8 T cells from tumor-bearing mice failed to induce CD107a. e-g, Lm20 treated mice that completely cleared MC38 tumor or age-matched naïve controls were rechallenged with MC38 after being tumor free for: CR\#1:198 days, CR\#2: 100 days, CR\#3: 216 days. e, flow cytometric phenotyping of circulating $T$ cells in peripheral blood from CR\#2 before tumor implantation (top panel) and 7 days post re-challenge (bottom panel). f, Ex vivo IFN $\gamma^{+}$spots circulating in the peripheral blood from CR\#2 before and after MC38 re-challenge, re-stimulated with minimal peptides from Lm20 validated neoantigens in pools of 4, or 9mer of MT Adpgk and Copg1 alone. Pooling detailed in online supplementary table 2. $\mathbf{g}$, Tumor volume kinetics and survival in MC38 re-challenged mice. Statistics; a, Tumor volume, measurement of Area Under Curve, One way ANOVA, ***, $\mathrm{P}=0.0006$; Survival curve, Log-rank (Mantel-Cox) test, $* * *, P=0.0004$. f, Unpaired t-test, $*, 0.01<\mathrm{P}<0.05$; **, $0.001<\mathrm{P}<0.01 ; * * *, 0.0001<\mathrm{P}<0.001 ; * * * *, \mathrm{P}<0.0001$. g, Two-way ANOVA and Tukey multiple comparison*, $0.01<\mathrm{P}<0.05$; $* *, 0.001<\mathrm{P}<0.01$.; Survival analysis Log-rank test $*, 0.01<\mathrm{P}<0.05$. 
a
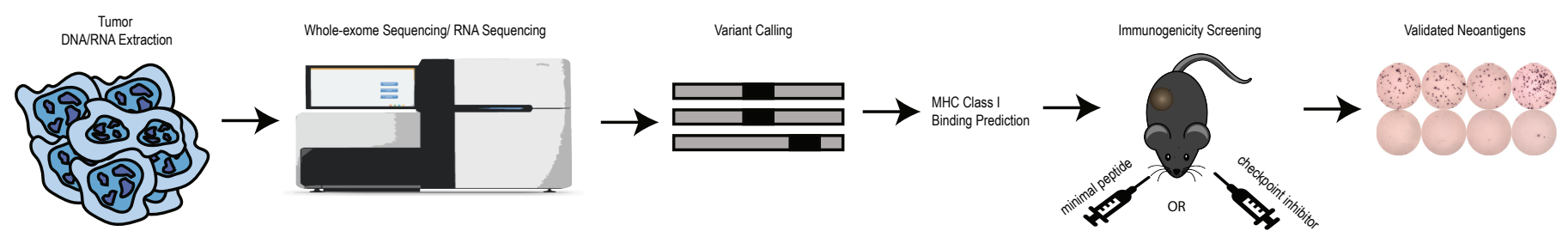

b
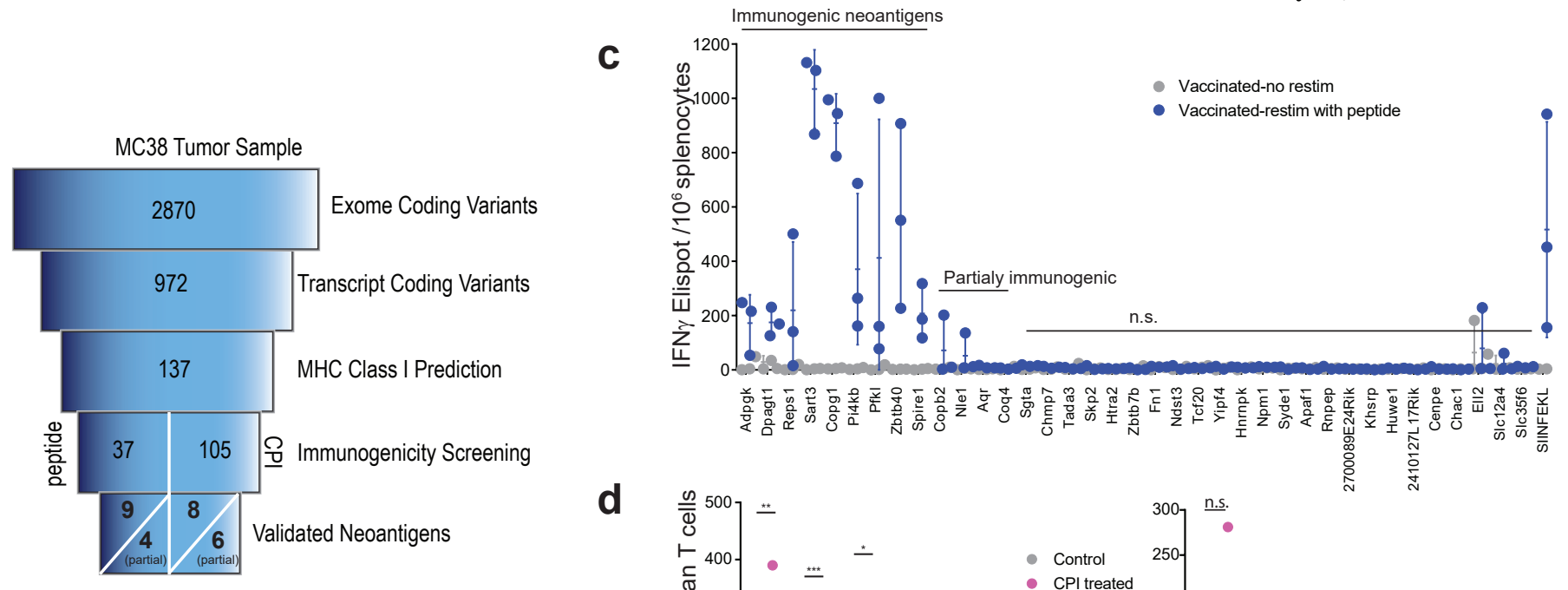

e

d
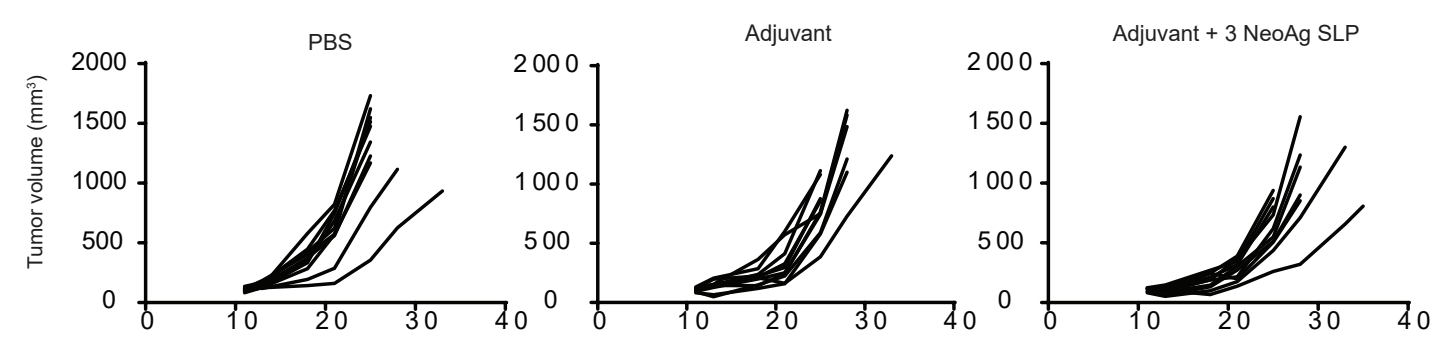

\begin{tabular}{rl|l} 
n.s.| & - PBS \\
n.s. & + Adjuvant \\
\hline & Adjuvant+
\end{tabular}

n.s. - Adjuvant+ 3 NeoAg SLP
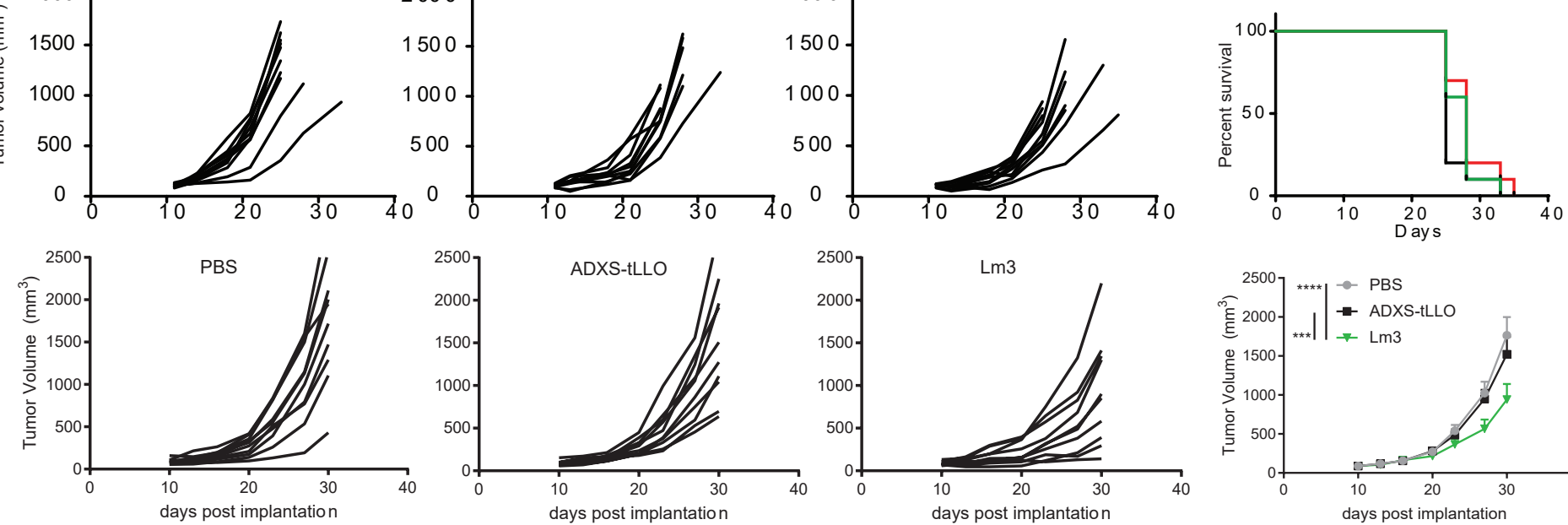

g

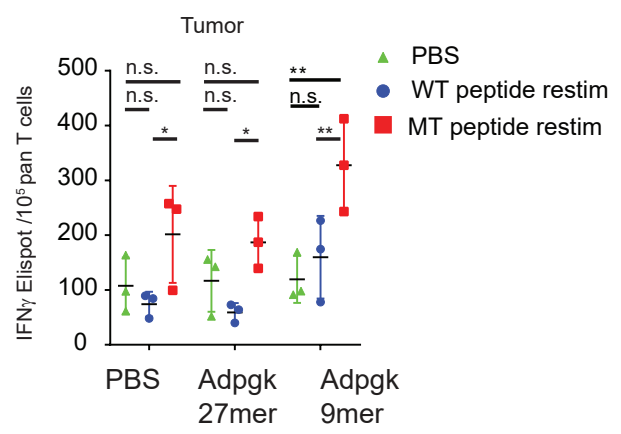

h

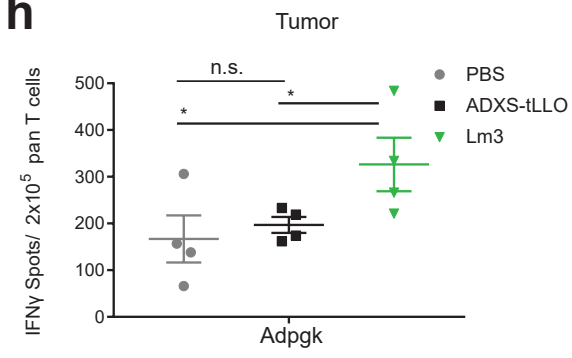

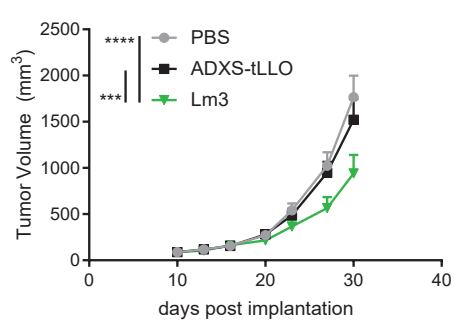

i

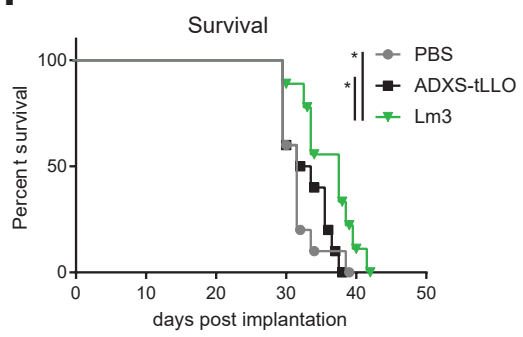


A

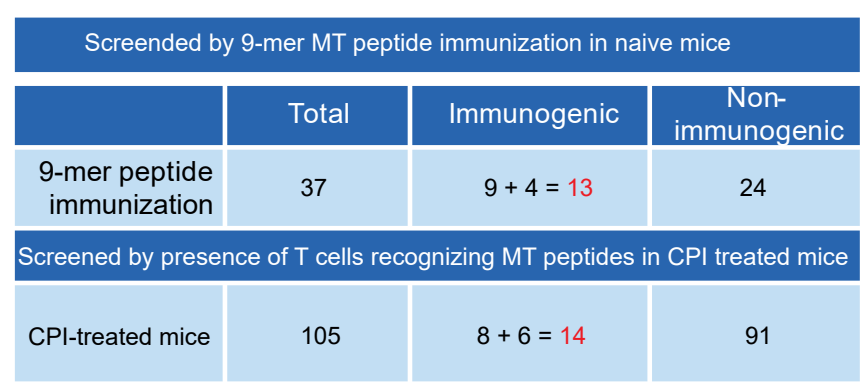

\section{C}

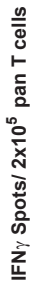

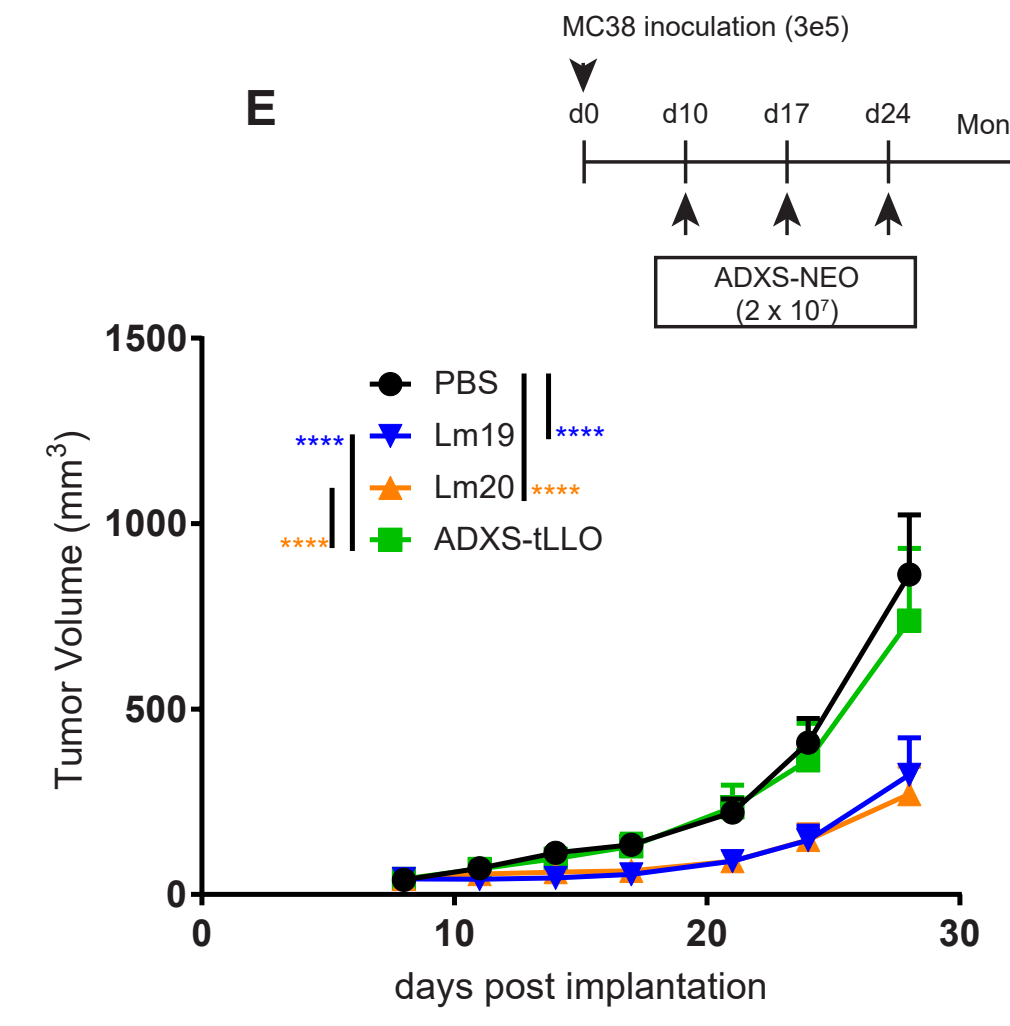

MC38 inoculation (3e5)

B Beads on a string construct design

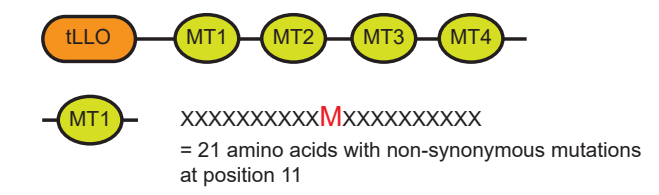

Lm19 : 19 screened NSMs that failed to elicit a positive IFN $\gamma$ ELISpot

Lm20 : 11 screended NSMs that generated positive IFN $\gamma$ spots + 9 NSMs that generated IFN $\gamma$ in MC38 tumor-bearing mice treated with checkpoint inhibitor

Figure 2
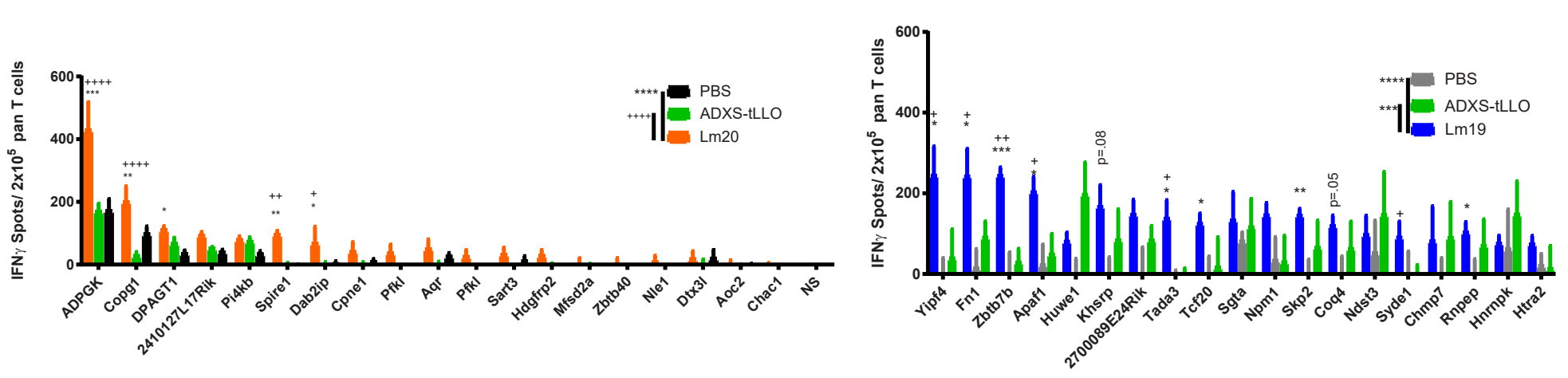

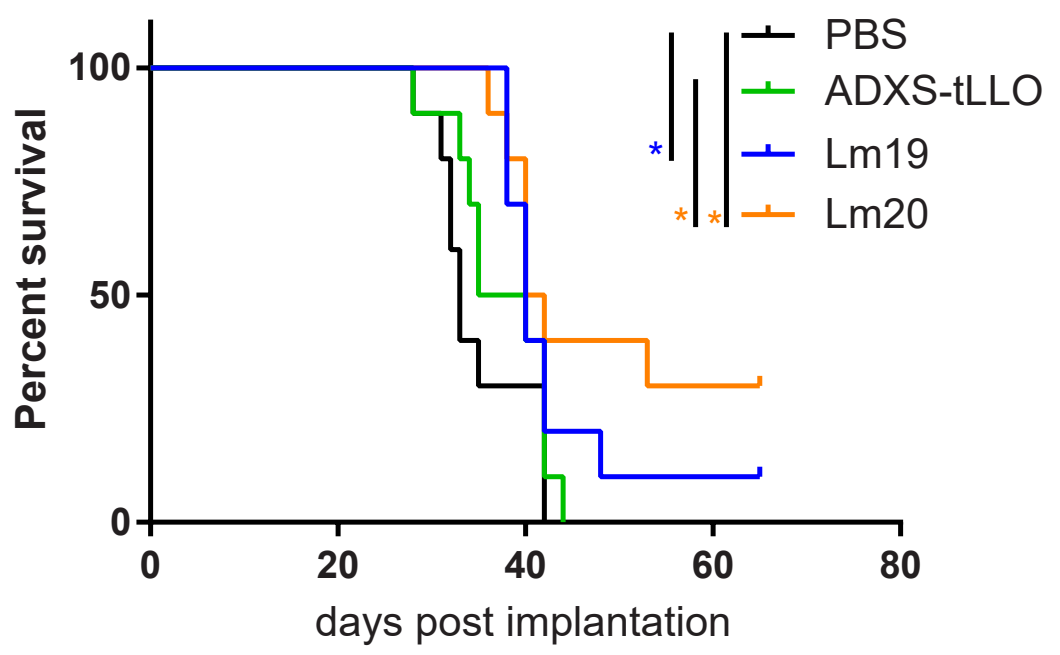


a

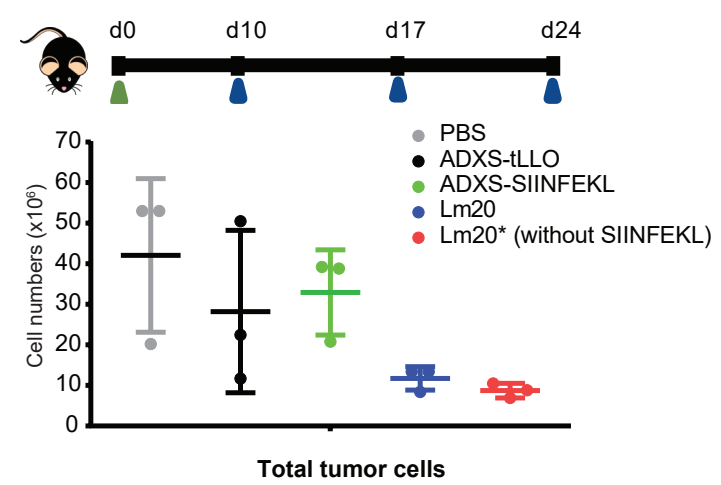

Figure 3

b

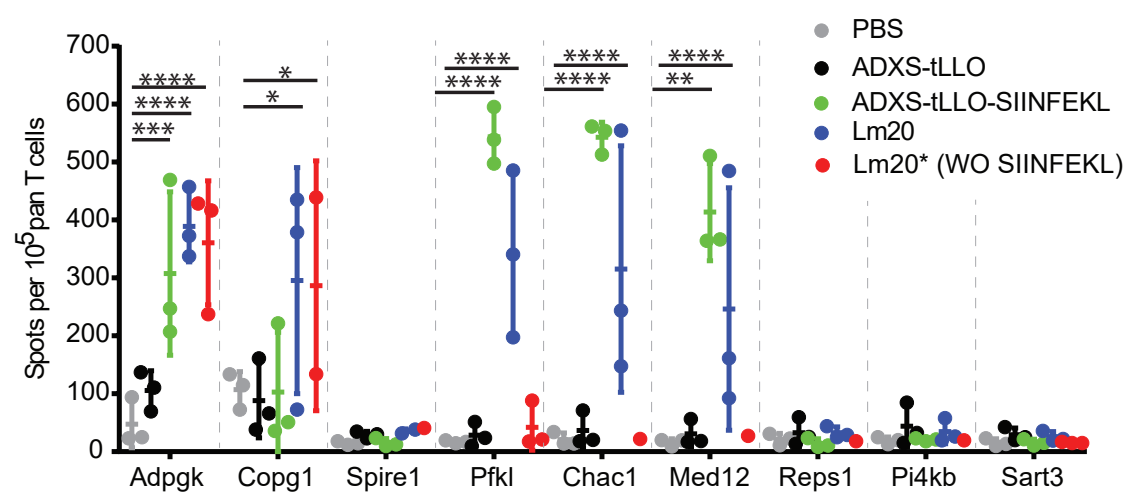

C

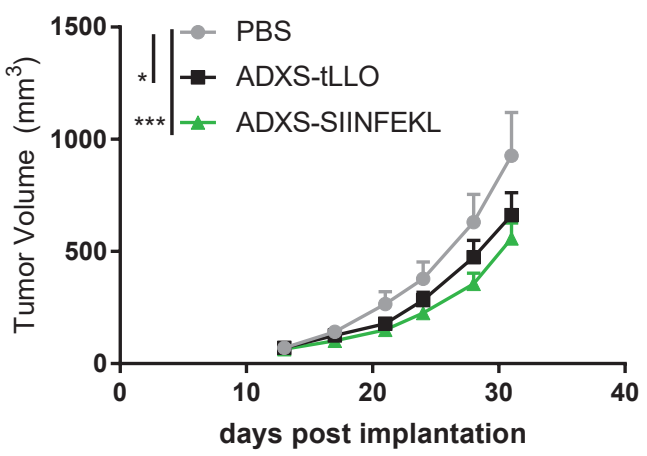


bioRxiv preprint doi: https://doi.org/10.1101/2020.05.11.088930; this version posted May 13, 2020. The copyright holder for this preprint (which was not certified by peer review) is the author/funder. All rights reserved. No reuse allowed without permission.

a

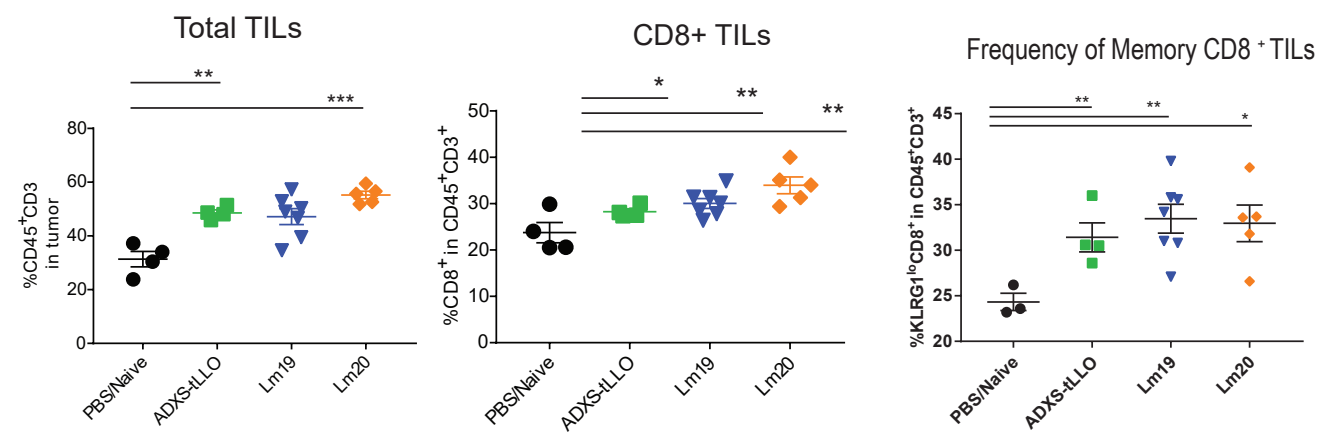

Figure 4

b

CD8+ Effectors in Tumor

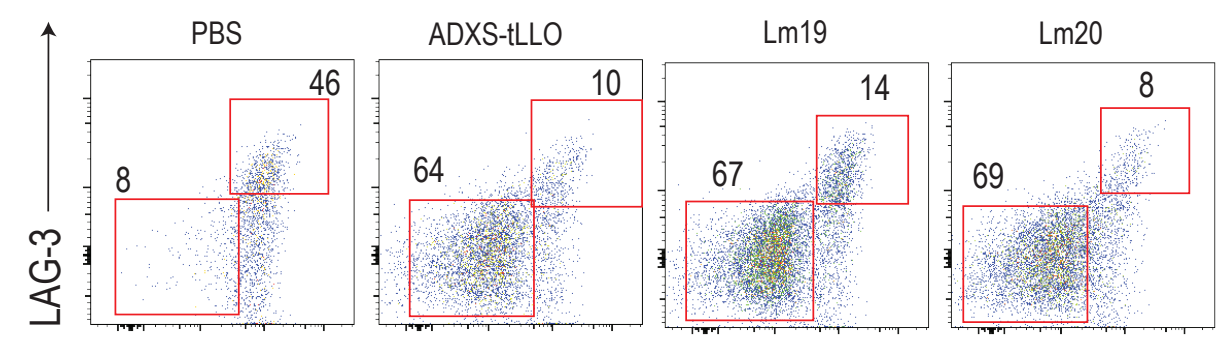

PD-1

C

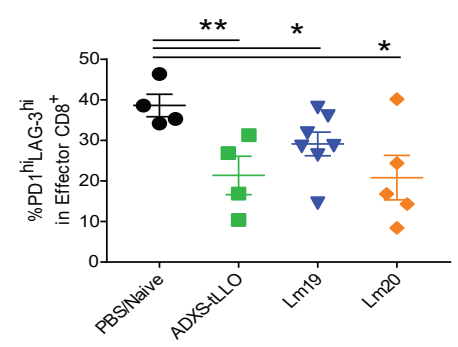

g

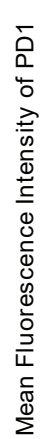

d (exhausted vs PD $1^{10}$ effector CD8 TILs)

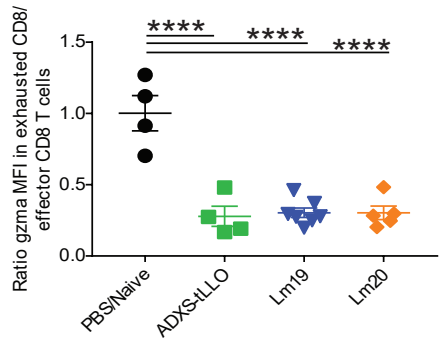

h

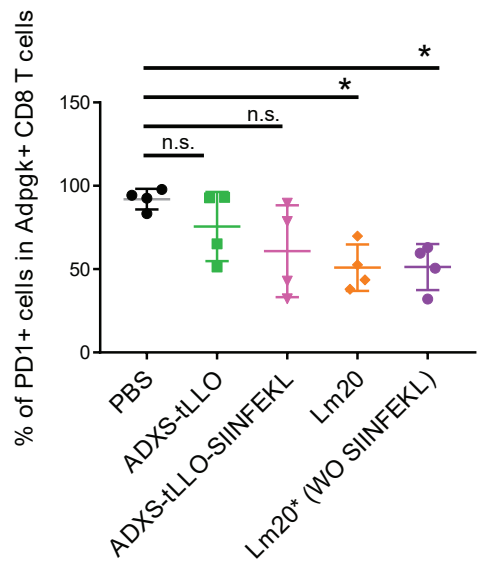

f

Frequency of PD-1 ${ }^{10}$ effector CD8 TILS

Frequency of Cytotoxic PD-1 ${ }^{10}$ Effector CD8 TILs

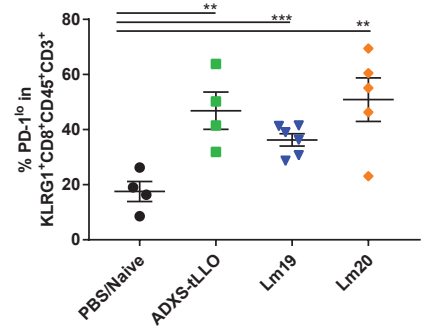

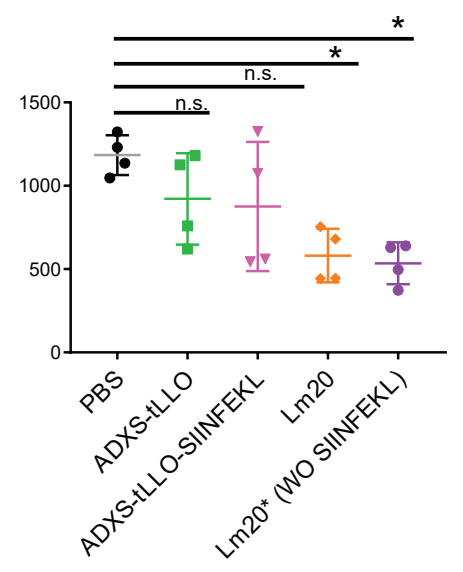


bioRxiv preprint doi: https://doi.org/10.1101/2020.05.11.088930; this version posted May 13, 2020. The copyright holder for this preprint (which was not certified by peer review) is the author/funder. All rights reserved. No reuse allowed without permission.

a

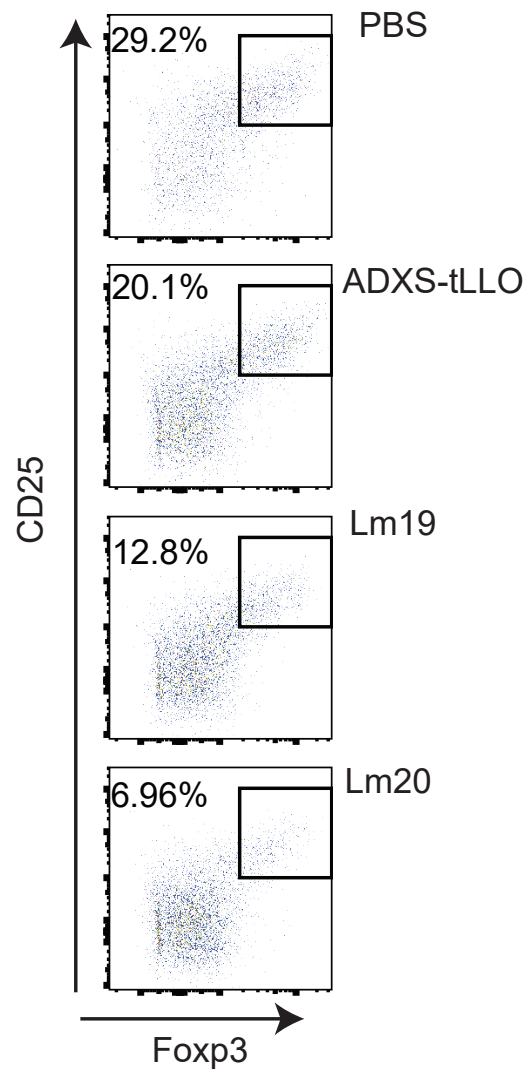

b Frequency Intratumoral Tregs

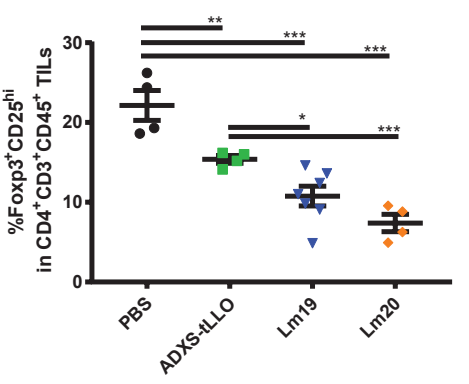

C

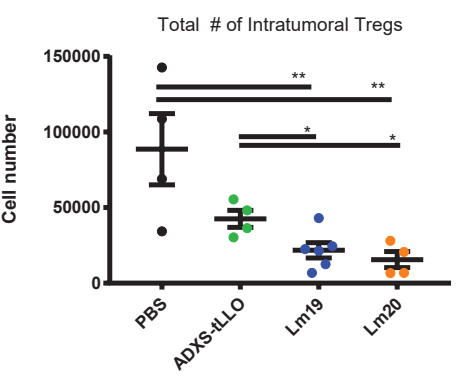

d

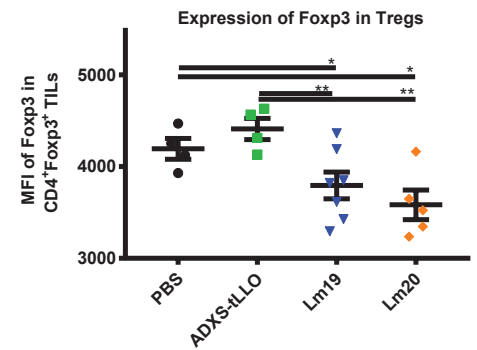

\section{d}

M1/M2 macrophages in TME

e

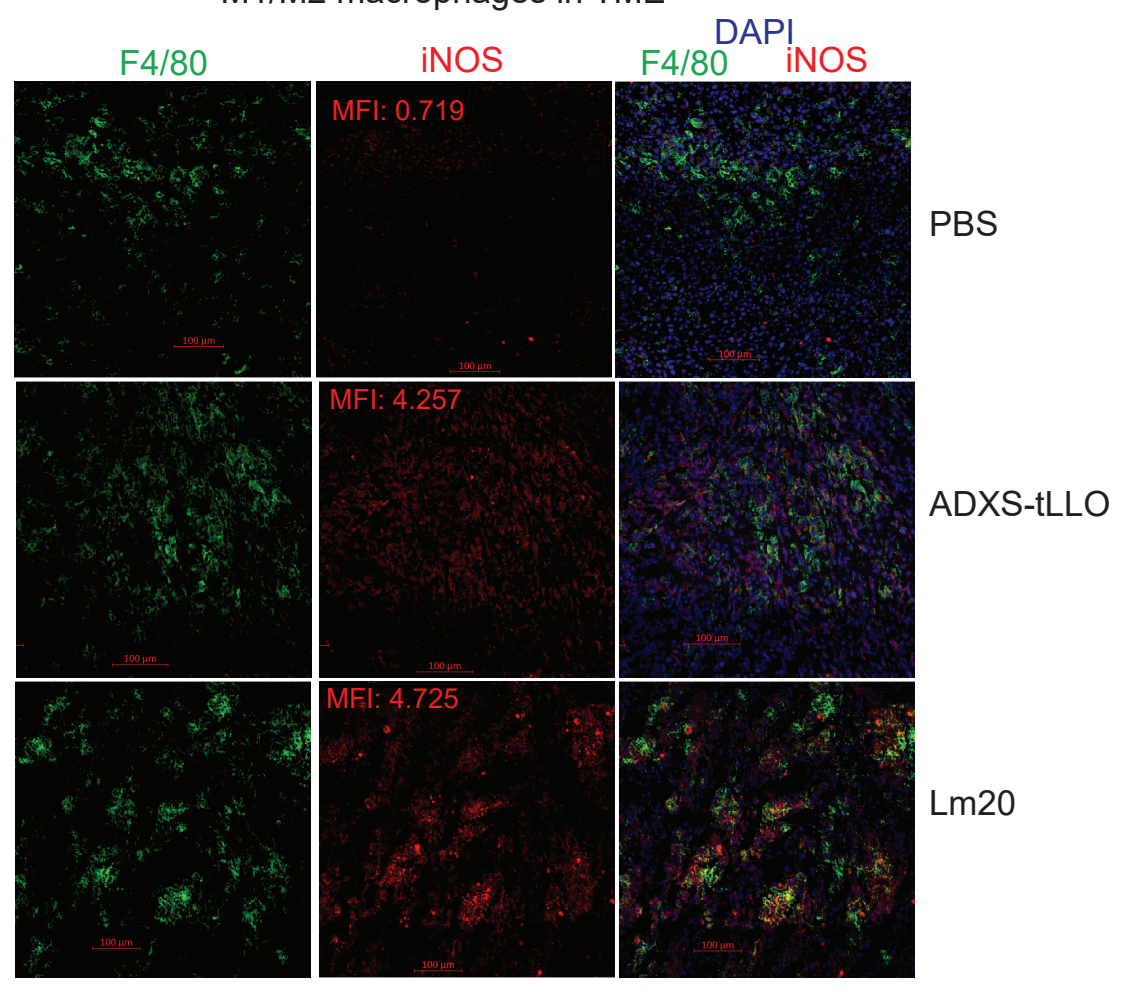

$\mathrm{F} 4 / 80$

ARG-1

DAPI

F4/80 ARG-1
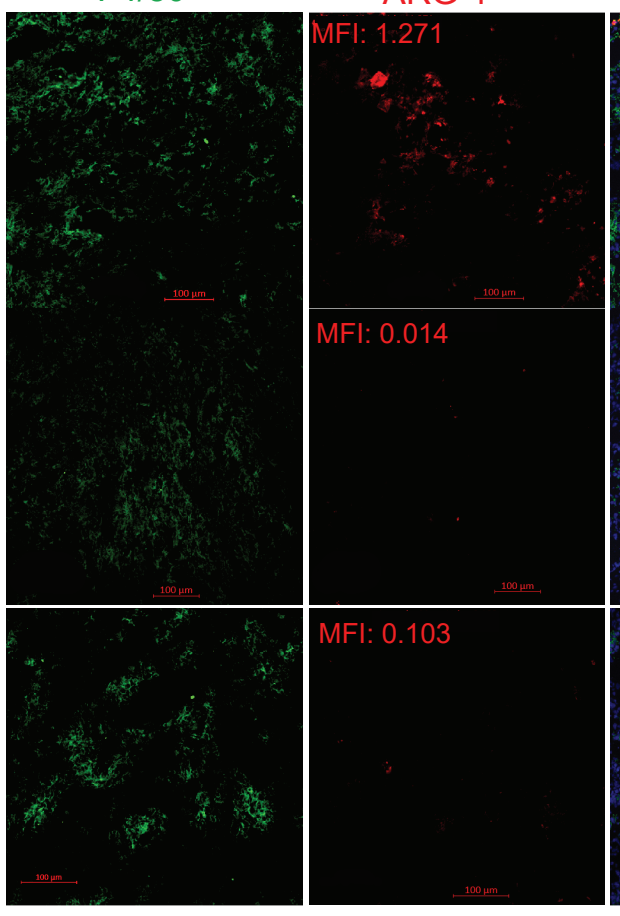

Figure 5 
a
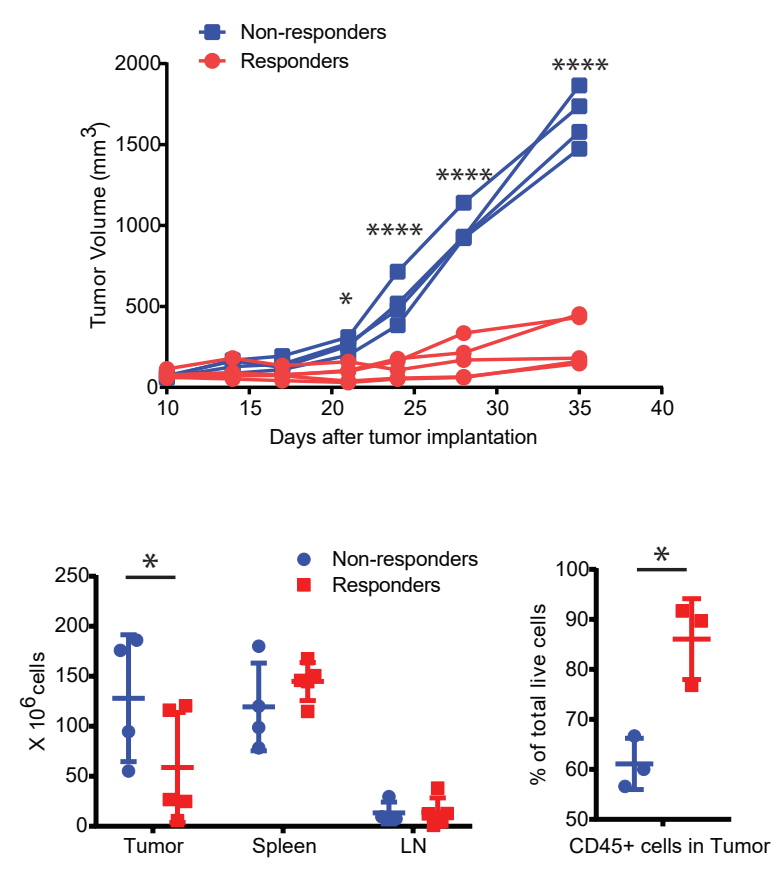

C

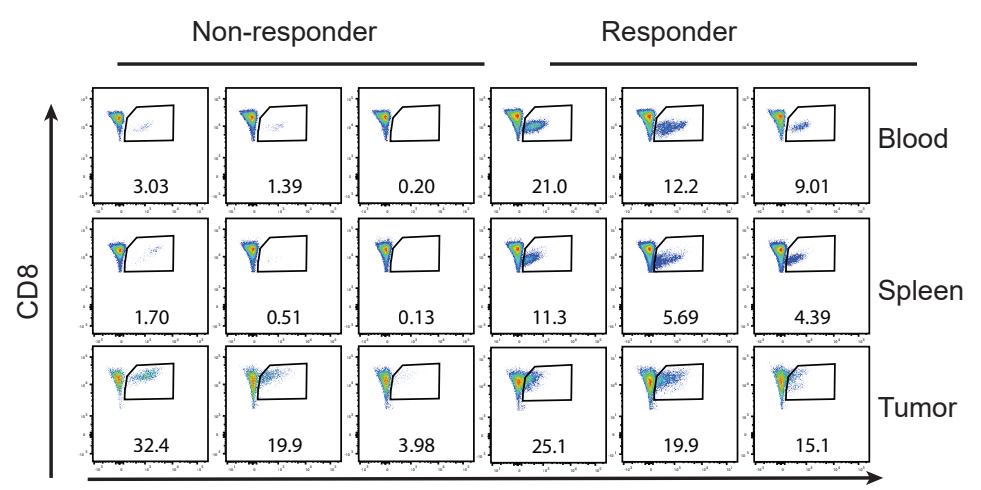

Adpgk Dextramer

\section{Figure 6}

\section{b}

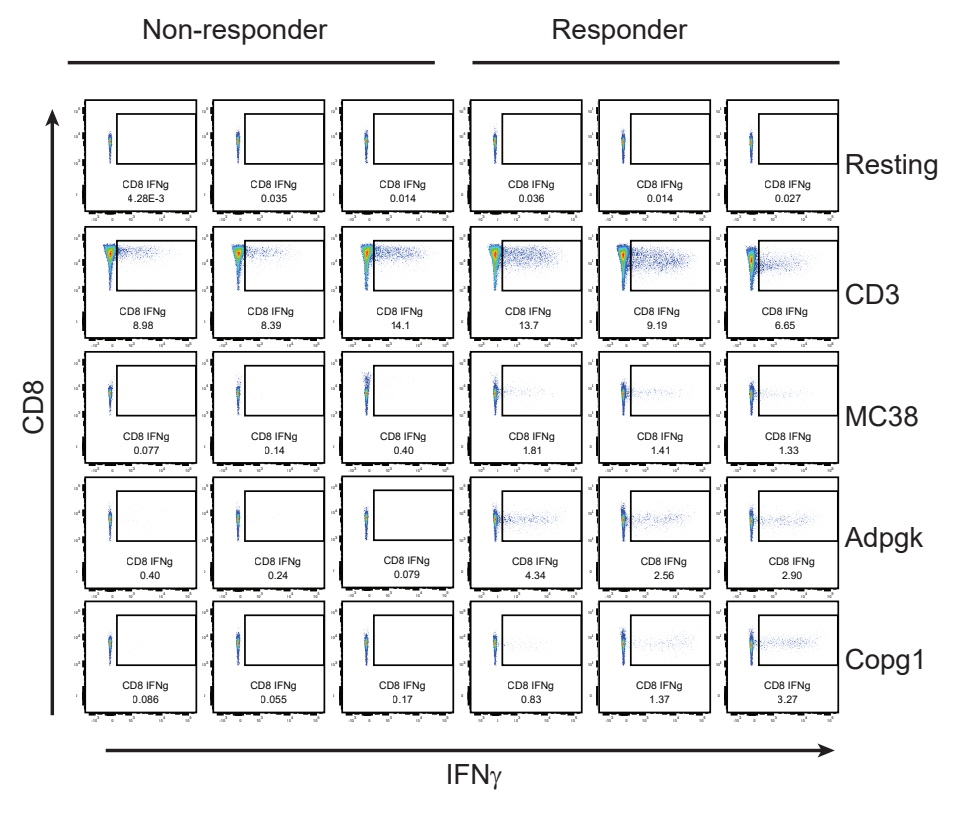

d

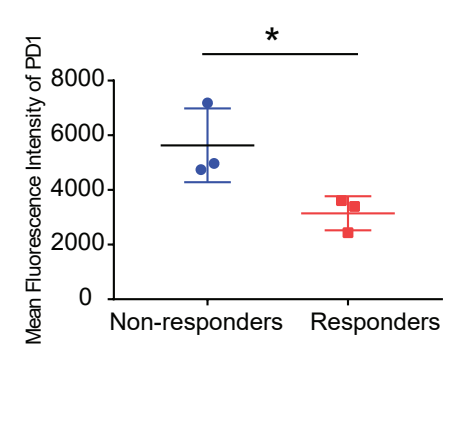

e

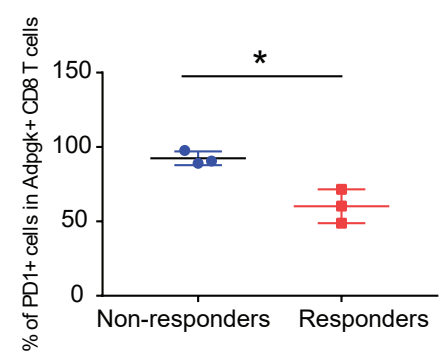


bioRxiv preprint doi: https://doi.org/10.1101/2020.05.11.088930; this version posted May 13, 2020. The copyright holder for this preprint (which was not certified by peer review) is the author/funder. All rights reserved. No reuse allowed without permission.

a

MC38 inoculation $\left(3 \times 10^{5}\right)$
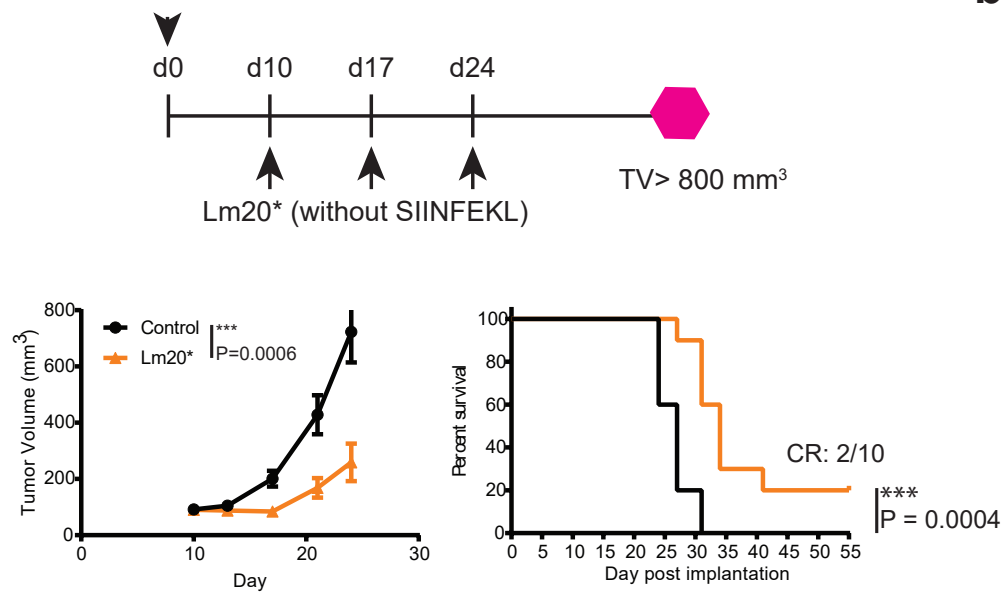

C

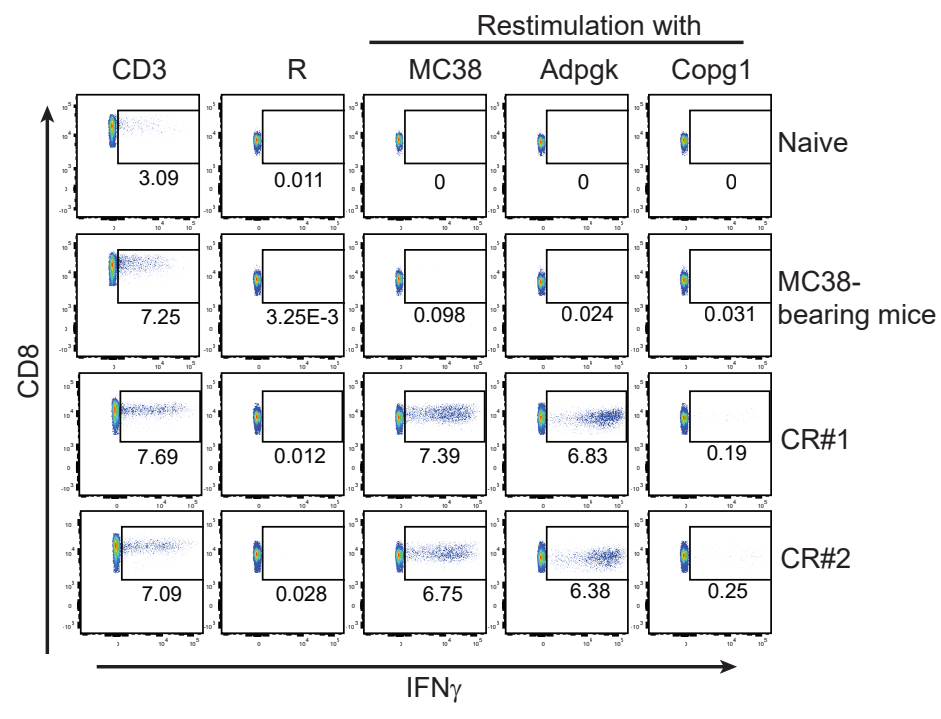

e

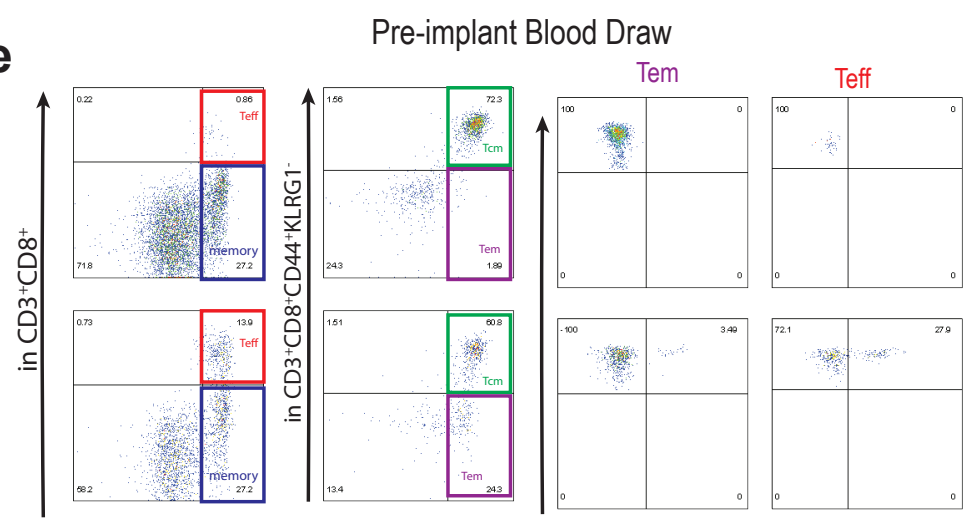

Day 7 Post-Re-challenge
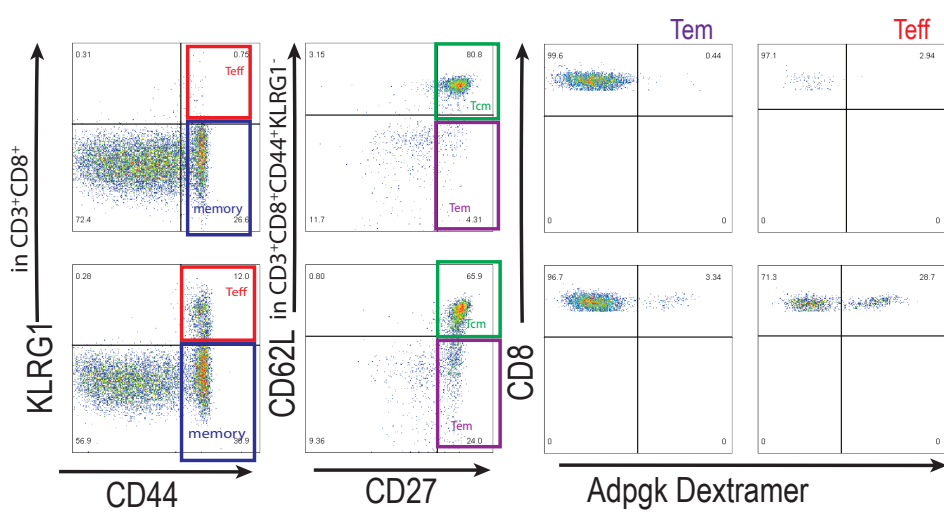

b

Figure 7

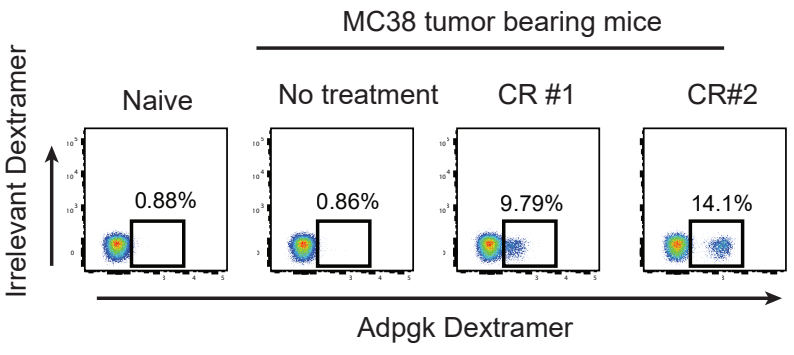

d

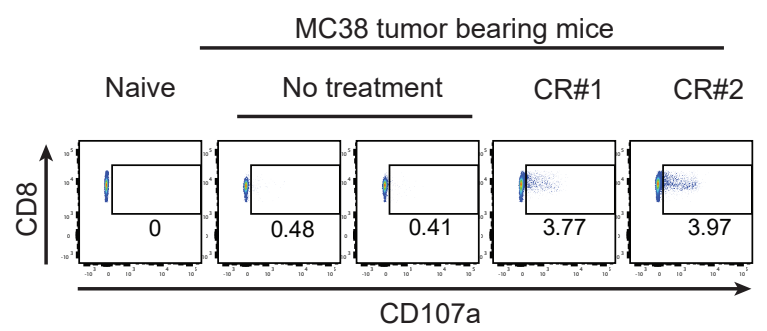

CR

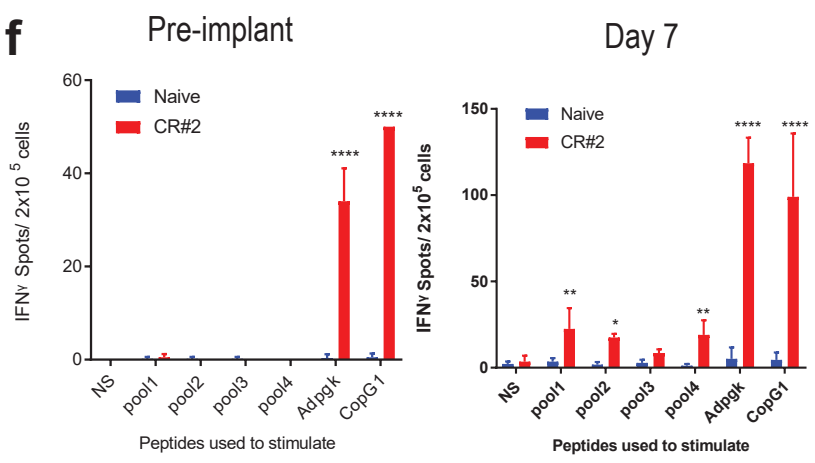

Day 100

PBS
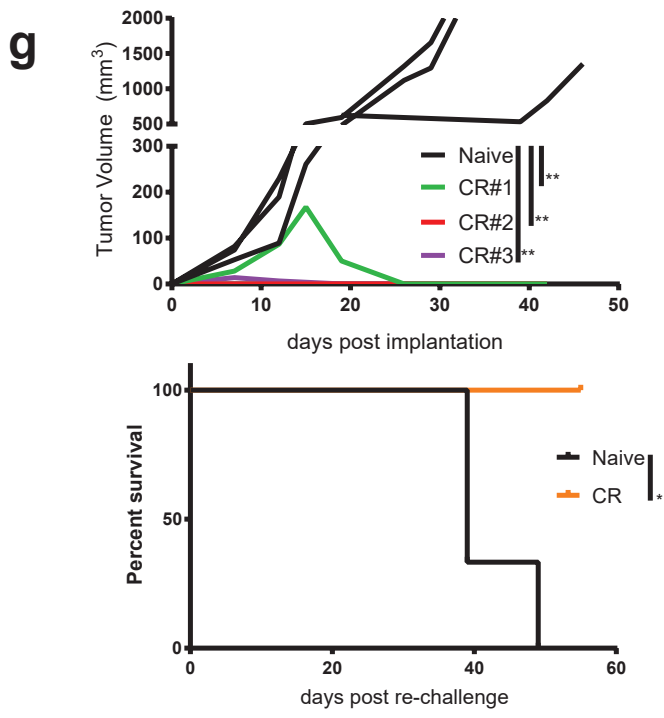